\title{
CAMA
}

Centre for Applied Macroeconomic Analysis

\section{The (Ir)Relevance of Rule-of-Thumb Consumers for US Business Cycle Fluctuations}

\section{CAMA Working Paper 102/2020 November 2020}

\author{
Alice Albonico \\ University of Milano - Bicocca \\ CefES
}

\section{Guido Ascari}

University of Oxford

University of Pavia

\section{Qazi Haque}

University of Western Australia

Centre for Applied Macroeconomic Analysis, ANU

\section{Abstract}

We estimate a medium-scale model with and without rule-of-thumb consumers over the preVolcker and the Great Moderation periods, allowing for indeterminacy. Passive monetary policy and sunspot fluctuations characterize the pre-Volcker period for both models. The estimated fraction of rule-of-thumb consumers is low, such that the models are empirically almost equivalent. In both subsamples, the two models yield very similar impulse response functions, variance and historical decompositions. We conclude that rule-of-thumb consumers are irrelevant to explain aggregate U.S. business cycle fluctuations. 


\title{
Keywords
}

rule-of-thumb consumers, indeterminacy, business cycle fluctuations

\author{
JEL Classification
}

E32, E52, C11, C13

Address for correspondence:

(E) cama.admin@anu.edu.au

ISSN 2206-0332

The Centre for Applied Macroeconomic Analysis in the Crawford School of Public Policy has been established to build strong links between professional macroeconomists. It provides a forum for quality macroeconomic research and discussion of policy issues between academia, government and the private sector.

The Crawford School of Public Policy is the Australian National University's public policy school, serving and influencing Australia, Asia and the Pacific through advanced policy research, graduate and executive education, and policy impact. 


\title{
The (Ir)Relevance of Rule-of-Thumb Consumers for U.S. Business Cycle Fluctuations*
}

\author{
Alice Albonico ${ }^{\dagger}$ \\ University of Milano - Bicocca \\ CefES
}

\author{
Guido Ascari ${ }^{\ddagger}$ \\ University of Oxford \\ University of Pavia
}

\author{
Qazi Haque ${ }^{\S}$ \\ University of Western Australia \\ Centre for Applied Macroeconomic Analysis
}

November 11, 2020

\begin{abstract}
We estimate a medium-scale model with and without rule-of-thumb consumers over the pre-Volcker and the Great Moderation periods, allowing for indeterminacy. Passive monetary policy and sunspot fluctuations characterize the pre-Volcker period for both models. The estimated fraction of rule-of-thumb consumers is low, such that the models are empirically almost equivalent. In both subsamples, the two models yield very similar impulse response functions, variance and historical decompositions. We conclude that rule-of-thumb consumers are irrelevant to explain aggregate U.S. business cycle fluctuations.
\end{abstract}

Keywords: rule-of-thumb consumers, indeterminacy, business cycle fluctuations JEL classification: E32, E52, C11, C13

${ }^{*}$ We thank Andrea Colciago and Chiara Punzo for comments. We also thank seminar participants at University of Milano - Bicocca. Haque acknowledges generous support from the Australian Research Council, under the grant DP170100697.

${ }^{\dagger}$ Address: Department of Economics, Management and Statistics, University of Milano - Bicocca, Piazza dell'Ateneo Nuovo 1, 20126 Milano, Italy. Phone: +390264483041. Email: alice.albonico@unimib.it

$\ddagger$ Address: Department of Economics, University of Oxford, Manor Road, Oxford OX1 3UQ, United Kingdom. Email: gudo.ascari@economics.ox.ac.uk

$\S$ Address: Department of Economics, The University of Western Australia, 35 Stirling Hwy, Crawley WA6009, Australia. Email: qazi.haque@uwa.edu.au. 


\section{Introduction}

In the last two decades, a growing body of literature introduced some dimensions of agents' heterogeneity in macroeconomic models. This started with simple Two-Agents New-Keynesian (TANK) models and evolved with the more complex Heterogeneous-Agents (HANK) models, following Kaplan et al. (2018). Among TANK model specifications, many papers consider the presence of the so-called Rule-of-Thumb (ROT, henceforth) consumers. In line with the seminal papers by Galí et al. (2007) and Bilbiie (2008), these are liquidity constrained households who cannot access financial and capital markets and thus cannot smooth consumption. This feature enables to move from the standard Representative-Agent (RANK) specification while keeping the model tractable from an analytical point of view. The presence of ROT consumers proved to be beneficial for New Keynesian models aiming at reproducing empirical dynamics in response to government spending shocks (Galí et al., 2007), investment shocks (Furlanetto et al., 2013) and technology shocks (Furlanetto and Seneca, 2012). From a theoretical point of view, ROT models can alter monetary and fiscal policy prescriptions. This is extensively analyzed by Bilbiie (2008), where he finds the occurrence of an Inverted Aggregate Demand Logic (IADL) leading to an upward sloping AD curve for a high enough share of ROT.

The ROT assumption has been introduced in estimated macroeconomic models. Kaplan et al. (2014), among others, show that liquidity constrained agents could be relevant empirically. Nowadays, important institutions such as the Federal Reserve (Brayton et al., 2014) and the European Commission (Kollmann et al., 2016) are including this type of agents in their benchmark estimated models used for forecasting and for the analysis of macroeconomic issues. Coenen at al. (2012), Forni et al. (2009) and Albonico et al. (2019), among others, estimate medium-scale DSGE models with ROT for the Euro area. For the U.S., the literature focuses more on standard representative agents models such as Smets and Wouters (2007), which is now the benchmark for estimated models for the U.S. economy. A notable exception is Bilbiie and Straub (2013), where the authors estimate a simple DSGE model to study the Great Inflation and the Great Moderation periods in the U.S.. Until then, some renowned works in the literature ascribed the occurrence of the Great Inflation episode to "bad policy" of the Federal Reserve, nevertheless failing to provide an explanation for the change in Fed's behavior. Clarida et al. (2000) point toward self-fulfilling expectations due to indeterminacy 
arising from passive monetary policy as an explanation of the high inflation episode in the U.S. during the 1970s. Following their suggestion, Lubik and Schorfheide (2004) propose a method to quantitatively assess the importance of equilibrium indeterminacy and the propagation of fundamental and sunspot shocks in this case. Bilbiie and Straub (2013) put forward an alternative explanation of the Great Inflation episode arguing that the different monetary policy transmission mechanisms which characterized those periods could be related to a structural change in asset market participation. Precisely, they find that between the pre-Volcker and the Volcker-Greenspan subsamples, the fraction of liquidity constrained agents decreased and monetary policy changed from passive to active. Due to the IADL mechanism, however, the equilibrium always stays determinate.

The recent developments of HANK models pose some questions about the relevance of heterogeneity on aggregate fluctuations. Debortoli and Galí (2017) compare the implications for business cycles fluctuations between a HANK model and a simpler TANK model with ROT consumers. Identifying the three sources of heterogeneity arising in the HANK framework ${ }^{1}$, they show that the most important component of heterogeneity for output fluctuations is the consumption gap between the two types of consumers (constrained and unconstrained). Interestingly, they show that a simple TANK model, with a constant share of constrained households and no heterogeneity within either type, approximates the implications of a HANK model regarding output fluctuations reasonably well, thereby supporting the use of a TANK model in quantitative analysis of U.S. business cycle fluctuations. Along these lines, Bayer et al. (2020) estimate a medium-scale HANK model and show that adding data on inequality does not affect aggregate fluctuations in the U.S. Moreover, using survey data from the U.S. Survey of Consumer Finances, Kaplan et al. (2014) measure the fraction of the so-called poor Hand-to-Mouth consumers $^{2}$ to be $14 \%$ on average in the U.S. between 1989 and 2010.

In this paper, we investigate the relevance of ROT consumers in explaining U.S. business cycle fluctuations, revisiting the findings of Bilbiie and Straub (2013). We introduce the presence of ROT consumers in a medium-scale DSGE model with all the standard bells and whistles similar to Smets and Wouters (2007). We then estimate the model over two different

\footnotetext{
${ }^{1}$ Namely, i) changes in the average consumption gap between constrained and unconstrained households, ii) variations in consumption dispersion within unconstrained households, and iii) changes in the share of constrained households. See also Bilbiie (2020) for related important work on the comparison between HANK and TANK.

${ }^{2}$ Poor Hand-to-Mouth consumers are similar to ROT consumers.
} 
subsamples (the pre-Volcker and the Great Moderation periods), while allowing and testing for (in)determinacy, and compare our results with the standard RANK specification. In this context, indeterminacy can arise due to different combinations of parameters. For instance, for low values of the degree of ROT, indeterminacy can arise due to passive monetary policy, dubbed the Standard Aggregate Demand Logic (SADL), as in Lubik and Schorfheide (2004). In contrast, for high enough values of the degree of ROT share, an IADL might be in place as in Bilbiie (2008), resulting in either indeterminacy due to active monetary policy or determinacy if monetary policy is passive, as found by Bilbiie and Straub (2013). Our paper is also related to Nicolò (2020), who estimates the model of Smets and Wouters (2007) for different subsamples while allowing for indeterminacy and employing the methodology proposed by Bianchi and Nicolò (2019). He shows that monetary policy was passive in the Great Inflation period and active afterwards. Similar to Lubik and Schorfheide (2004), he finds that indeterminacy manifested primarily by altering the propagation of structural shocks, while sunspot shocks played only a limited role in explaining macroeconomic volatility.

We find that introducing ROT consumers in a medium-scale model is irrelevant to explain aggregate business cycle fluctuations in U.S. data. The reason is that the estimated fraction of ROT consumers is so low that it is not affecting the dynamics of the model compared to a standard representative agent model (RANK). First, the estimations of both a model with ROT and one without (RANK) point to an indeterminate equilibrium in the pre-Volcker period, due to passive monetary policy, and to a determinate equilibrium in the post-Volcker period with active monetary policy, as in Lubik and Schorfheide (2004) or Nicolò (2020). Second, in the preVolcker period the log-likelihoods of the two models are very close, while in the latter period the RANK model is preferred by the data. Third, in both subsamples, the RANK and ROT models yield almost the same impulse response functions, variance and historical decompositions, such that they share the same narrative of U.S. business cycle fluctuations. Therefore, the presence of ROT consumers is not substantive to explain these fluctuations. The estimation results of the empirically rich medium-scale New Keynesian model therefore contrast with the ones in Bilbiie and Straub (2013), who employ a small-scale model.

As such, our results point toward the irrelevance of ROT consumers and imply that a medium-scale RANK model, like Smets and Wouters (2007), does not need to be enlarged by 
the presence of ROT to study the drivers of U.S. business cycle fluctuations. Nevertheless, this does not mean that modelling ROT, or heterogeneous agents more generally, is not important to explain other dimensions of the data, or that shocks and policy interventions affect different types of agents evenly. ${ }^{3}$ For instance, Bayer et al. (2020) show that the estimated shocks from their HANK model have significantly contributed to the evolution of U.S. wealth and income inequality.

The paper is organized as follows. Section 2 briefly presents the model. Section 3 explains the estimation strategy based on Bianchi and Nicolò (2019). Section 4 provides the main results and some robustness, while Section 5 concludes.

\section{Model}

We develop a Dynamic Stochastic General Equilibrium (DSGE) model following Smets and Wouters (2007) in particular. Smets and Wouters's (2007) model has become the workhorse model for the empirical analysis of the U.S. economy. It includes all the standard features and frictions of New-Keynesian models, while still remaining tractable. We depart from their model only in few aspects. First, we introduce the presence of Rule-of-Thumb (ROT) consumers, on the footsteps of Galí et al. (2007) and Bilbiie (2008). There is a fraction $\theta$ of households who do not have access to financial and capital markets and consume all their disposable labor income in each period. Second, we consider a separable utility function in consumption and hours, to stay close to Bilbiie and Straub $(2012,2013)$. Wage decisions are made by unions which optimally reset the nominal wage according to a Calvo (1983) scheme. The supply side is composed of final producers operating under perfect competition and intermediate monopolistically competitive firms. Prices are sticky following a Calvo (1983) mechanism. Intermediate goods are packed by final firms with a Kimball (1995) aggregator.

The model includes the usual frictions considered in New-Keynesian medium-scale models: external habits in consumption, variable capital utilization, investment adjustment costs, sticky

\footnotetext{
${ }^{3}$ Most of the existing work concentrates on the effects of monetary policy shocks, which, however, do not play a prominent role in our variance and historical decompositions. See Colciago et al. (2019) for a survey of the literature about the effects of monetary policy on inequality. Findings are mixed, depending also on the type (conventional vs unconventional) of policy intervention. Slacalek et al. (2020) estimate a structural VAR for the euro area to quantify the different transmission mechanisms of monetary policy on household consumption expenditures.
} 
wages and prices, indexation on past and trend inflation.

Given that the model is rather standard, we leave a more detailed description of the model equations in the Appendix.

\subsection{Households}

There is a continuum of households indexed by $i \in[0,1]$. A share $1-\theta$ of households are Ricardian $(i=o)$, such that they can access financial markets, hold government bonds, accumulate physical capital, and rent capital services to firms. The remaining $\theta$ households are ROT consumers $(i=r t)$, as specified above.

Households maximize the following utility function:

$$
E_{0} \sum_{t=0}^{\infty} \beta^{t}\left\{\frac{1}{1-\sigma}\left(c_{t}^{i}-b c_{t-1}\right)^{1-\sigma}-\frac{\left(h_{t}^{i}\right)^{1+\phi_{l}}}{1+\phi_{l}}\right\}
$$

where individual and aggregate consumption $\left(c_{t}^{i}, c_{t}\right)$ are adjusted by the deterministic growth trend $g_{z}, h_{t}^{i}$ stands for individual hours worked, $0<\beta<1$ is the subjective discount factor, $\sigma$ measures the inverse of the intertemporal elasticity of substitution and $\phi_{l}$ is the inverse of Frisch elasticity. The parameter $0<b<1$ measures the degree of external habits in consumption.

Ricardian households budget constraint is standard:

$$
P_{t} C_{t}^{o}+P_{t} I_{t}^{o}+\frac{B_{t+1}^{o}}{\varepsilon_{t}^{b}}=R_{t-1} B_{t}^{o}+W_{t} h_{t}^{o}+P_{t} D_{t}^{o}+\left[R_{t}^{k} u_{t}^{o}-a\left(u_{t}^{o}\right) P_{t}\right] K_{t}^{o}-T_{t}^{o}
$$

where $a\left(u_{t}^{o}\right)=\gamma_{u 1}\left(u_{t}^{o}-1\right)+\frac{\gamma_{u 2}}{2}\left(u_{t}^{o}-1\right)^{2}$ defines the capital utilization cost function, in line with Christiano at al. (2005). Ricardian households allocate their resources between consumption $C_{t}^{o}$, investments $I_{t}^{o}$ and government-issued bonds $B_{t}^{o}$. They receive income from labor services $W_{t} h_{t}^{o}$, from dividends $D_{t}^{o}$, from renting capital services $u_{t}^{o} K_{t}^{o}$ at the rate $R_{t}^{k}$ and from holding government bonds. $P_{t}$ is the aggregate price index, $R_{t}$ is the gross nominal interest rate, $K_{t}^{o}$ is the physical capital stock and $u_{t}^{o}$ defines capital utilization. $T_{t}^{o}$ are lump-sum taxes. $\varepsilon_{t}^{b}$ is a risk premium shock that affects the intertemporal margin, creating a wedge between the interest rate controlled by the central bank and the return on assets held by the households. 
The capital accumulation equation is:

$$
K_{t+1}^{o}=(1-\delta) K_{t}^{o}+\varepsilon_{t}^{i}\left[1-S\left(\frac{I_{t}^{o}}{I_{t-1}^{o}}\right)\right] I_{t}^{o}
$$

with the investment adjustment costs function defined as:

$$
S\left(\frac{I_{t}^{o}}{I_{t-1}^{o}}\right)=\frac{\gamma_{I}}{2}\left(\frac{I_{t}^{o}}{I_{t-1}^{o}}-g_{z}\right)^{2}
$$

where $\delta$ is the capital depreciation rate and $\gamma_{I}$ is a parameter measuring the degree of investment adjustment costs. $\varepsilon_{t}^{i}$ is a shock to the marginal efficiency of investment (see Justiniano et al., 2010).

ROT households maximize (1) subject to the following budget constraint:

$$
P_{t} C_{t}^{r t}=W_{t} h_{t}^{r t}
$$

A generic aggregate variable is expressed as $X_{t}=\theta X_{t}^{r t}+(1-\theta) X_{t}^{o}$.

\subsection{Labor market}

Each household supplies the bundle of labor services $h_{t}^{i}=\left\{\int_{0}^{1}\left[h_{t}^{i}(j)\right]^{\frac{1}{1+\lambda_{t}^{w}}} d j\right\}^{1+\lambda_{t}^{w}}$ that firms demand. For each labor type $j$, the wage setting decision is allocated to a specific labor union. At the given nominal wage $W_{t}^{j}$, households supply the amount of labor that firms demand. Following Colciago (2011), demand for labor type $j$ is split uniformly across the households, so that households supply identical amount of labor services, $h_{t}=h_{t}^{i}$. $\lambda_{t}^{w}$ represents an exogenous shock to the net wage markup.

\subsubsection{Wage setting}

Nominal wages are sticky à la Calvo (1983). In each period, union $j$ can optimally reset the nominal wage with probability $\left(1-\xi_{w}\right)$. Those unions that cannot re-optimize the wage adjust the wage according to the scheme $W_{t}^{j}=g_{z} \pi_{t-1}^{\chi_{w}} \pi^{\left(1-\chi_{w}\right)} W_{t-1}^{j}$, where $\pi$ is the steady state (or trend) inflation rate. Non-reset wages are partially indexed to past inflation and trend inflation, with $\chi_{w} \in[0,1]$ allowing for any degree of combination of indexation between the two 
components. The aggregate wage is thus:

$$
W_{t}=\left[\xi_{w}\left(g_{z} \pi_{t-1}^{\chi_{w}} \pi^{1-\chi_{w}} W_{t-1}\right)^{\frac{1}{\lambda_{t}^{w}}}+\left(1-\xi_{w}\right)\left(\tilde{W}_{t}\right)^{\frac{1}{\lambda_{t}^{w}}}\right]^{\lambda_{t}^{w}}
$$

where $\tilde{W}_{t}$ is the optimal reset wage.

Following Colciago (2011), we assume that the representative union's objective function is a weighted average $(1-\theta, \theta)$ of the two household types' utility functions, subject to the labor demand $h_{t}=h_{t}^{d} \int_{0}^{1}\left(\frac{W_{t}^{j}}{W_{t}}\right)^{-\frac{1+\lambda_{t}^{w}}{\lambda_{t}^{w}}} d j,(2)$ and (5). The resulting first order condition is:

$$
\begin{aligned}
& E_{t} \sum_{s=0}^{\infty}\left(\xi_{w} \beta\right)^{s} h_{t+s}^{j}\left\{\tilde{W}_{t}^{j} \frac{g_{z}^{s} \pi_{t, t+s-1}^{\chi_{w}} \pi^{s\left(1-\chi_{w}\right)}}{P_{t+s} g_{z}^{t+s}}\left(1-\frac{1+\lambda_{t}^{w}}{\lambda_{t}^{w}}\right)\left[\begin{array}{c}
(1-\theta)\left(c_{t+s}^{o}-b c_{t+s-1}\right)^{-\sigma} \\
+\theta\left(c_{t+s}^{r t}-b c_{t+s-1}\right)^{-\sigma}
\end{array}\right](7)\right. \\
& \left.+\frac{1+\lambda_{t}^{w}}{\lambda_{t}^{w}}\left[(1-\theta)\left(c_{t+s}^{o}-b c_{t+s-1}\right)^{-\sigma} M R S_{t+s}^{o}+\theta\left(c_{t+s}^{r t}-b c_{t+s-1}\right)^{-\sigma} M R S_{t+s}^{r t}\right]\right\}=0 .
\end{aligned}
$$

\subsection{Production}

\subsubsection{Final good firms}

The final good $Y_{t}$ is produced under perfect competition. A continuum of intermediate inputs $Y_{t}^{z}$ is combined as in Kimball (1995). The final good producers maximize profits:

$$
\begin{gathered}
\max _{Y_{t}, Y_{t}^{z}} P_{t} Y_{t}-\int_{0}^{1} P_{t}^{z} Y_{t}^{z} d z \\
\text { s.t. } \int_{0}^{1} G\left(\frac{Y_{t}^{z}}{Y_{t}} ; \lambda_{t}^{p}\right) d z=1,
\end{gathered}
$$

with $G$ strictly concave and increasing and $G(1)=1$ and $\lambda_{t}^{p}$ is the net price markup, which is assumed to be exogenous.

\subsubsection{Intermediate good firms.}

Intermediate firms $z$ are monopolistically competitive and use as inputs capital and labor services, $u_{t}^{z} K_{t}^{z}$ and $h_{t}^{z}$, respectively. The production technology is a Cobb-Douglas function $Y_{t}^{z}=\varepsilon_{t}^{a}\left[u_{t}^{z} K_{t}^{z}\right]^{\alpha}\left[g_{z}^{t} h_{t}^{z}\right]^{1-\alpha}-g_{z}^{t} \Phi$, where $\Phi$ are fixed production costs. $\varepsilon_{t}^{a}$ is a temporary total factor productivity shock. The term $g_{z}$ is a deterministic growth trend. 


\subsubsection{Price setting}

Intermediate goods prices are sticky à la Calvo (1983). A firm z can optimally reset its price with probability $\left(1-\xi_{p}\right)$. Firms that cannot re-optimize adjust the price according to the scheme $P_{t}^{z}=\pi_{t-1}^{\chi_{p}} \pi^{1-\chi_{p}} P_{t-1}^{z}$, where $\chi_{p} \in[0,1]$ allows for any degree of combination of indexation to past or trend inflation.

The aggregate price index is:

$$
P_{t}=\left(1-\xi_{p}\right) \tilde{P}_{t}^{z} G^{\prime-1}\left(\frac{\tilde{P}_{t}^{z} \iota_{t}}{P_{t}}\right)+\xi_{p} \pi_{t-1}^{\chi_{p}} \pi^{1-\chi_{p}} P_{t-1} G^{\prime-1}\left(\frac{\pi_{t-1}^{\chi_{p}} \pi^{1-\chi_{p}} P_{t-1} \iota_{t}}{P_{t}}\right)
$$

where $\iota_{t}=\int_{0}^{1} G^{\prime}\left(\frac{Y_{t}^{z}}{Y_{t}}\right) \frac{Y_{t}^{z}}{Y_{t}} d z$.

The representative firm chooses the optimal price $\tilde{P}_{t}^{z}$ that maximizes expected profits subject to the demand schedule. The resulting first order condition is:

$E_{t} \sum_{s=0}^{\infty} \xi_{p}^{s} \frac{\Xi_{t, t+s}}{P_{t+s}} Y_{t+s}^{z}\left[\tilde{P}_{t}^{z} \pi_{t, t+s-1}^{\chi_{p}} \pi^{s\left(1-\chi_{p}\right)}+\left(\tilde{P}_{t}^{z} \pi_{t, t+s-1}^{\chi_{p}} \pi^{s\left(1-\chi_{p}\right)}-M C_{t+s}^{z}\right) \frac{1}{G^{\prime-1}\left(\omega_{t+s}\right)} \frac{G^{\prime}\left(x_{t+s}\right)}{G^{\prime \prime}\left(x_{t+s}\right)}\right]=0$

where $\omega_{t}=\frac{\tilde{P}_{t}^{z}}{P_{t}} \iota_{t}$ and $x_{t}=G^{\prime-1}\left(\omega_{t}\right)$.

\subsection{Government}

The government budget constraint is:

$$
P_{t} G_{t}+R_{t-1} B_{t}=B_{t+1}+T_{t}
$$

We assume that it is balanced every period. Government spending evolves exogenously.

The monetary authority sets the nominal interest rate according to the same Taylor rule as in Smets and Wouters (2007):

$$
\frac{R_{t}}{R}=\left(\frac{R_{t-1}}{R}\right)^{\phi_{R}}\left[\left(\frac{\pi_{t}}{\pi}\right)^{\phi_{\pi}}\left(\frac{Y_{t}}{Y_{t}^{f l e x}}\right)^{\phi_{y}}\right]^{1-\phi_{R}}\left(\frac{Y_{t} / Y_{t-1}}{Y_{t}^{\text {flex }} / Y_{t-1}^{\text {flex }}}\right)^{\phi_{\Delta y}} \varepsilon_{t}^{r}
$$

where $Y_{t}^{\text {flex }}$ is the level of output prevailing in a flexible prices and wages environment and $\varepsilon_{t}^{r}$ is an exogenous interest rate shock. 


\section{Estimation strategy}

\subsection{Data}

To estimate the model, we use Bayesian techniques and the measurement equations that relate the macroeconomic data to the endogenous variables of the model are defined as:

$$
\left[\begin{array}{l}
d l G D P_{t} \\
d l C O N S_{t} \\
d l I N V_{t} \\
d l W A G_{t} \\
l H O U R S_{t} \\
d l P_{t} \\
F E D F U N D S_{t}
\end{array}\right]=\left[\begin{array}{l}
\bar{\gamma} \\
\bar{\gamma} \\
\bar{\gamma} \\
\bar{\gamma} \\
\bar{h} \\
\bar{\pi} \\
\bar{R}
\end{array}\right]+\left[\begin{array}{l}
\widehat{y}_{t}-\widehat{y}_{t-1} \\
\widehat{c}_{t}-\widehat{c}_{t-1} \\
\widehat{i}_{t}-\widehat{i}_{t-1} \\
\widehat{w}_{t}-\widehat{w}_{t-1} \\
\widehat{h}_{t} \\
\widehat{\pi}_{t} \\
\widehat{R}_{t}
\end{array}\right]
$$

where $d l$ denotes the percentage change measured as log difference, $l$ denotes the log, and hatted variables denote log deviations from steady state. The observables are the seven quarterly U.S. macroeconomic time series used in Smets and Wouters (2007), and they match the number of fundamental shocks that affect the economy. The series considered are: the growth rate in real GDP, consumption, investment and wages, log of hours worked, inflation rate measured by the GDP deflator, and the federal funds rate. Similar to Smets and Wouters (2007), $\bar{\gamma}$ denotes a deterministic growth trend common to the real variables GDP, consumption, investment and wages $\left(\bar{\gamma}=100\left(g_{z}-1\right)\right), \bar{h}$ is the (log) steady-state hours worked (normalized to zero), $\bar{\pi}$ is the quarterly steady-state net inflation rate, and $\bar{R}$ is the quarterly steady-state net nominal interest rate.

We include seven fundamental shock processes in the estimation (the same as in Smets and Wouters, 2007): a technology shock, a risk premium shock, an investment shock, a monetary policy shock, a government spending shock, a price markup shock and a wage markup shock. All shocks have an autoregressive component of order 1. The first four shocks are $\operatorname{AR}(1)$ processes with i.i.d. Normally distributed innovations. The government spending shock is also correlated with the technology shock. The two markup shocks also have a MA(1) component. 


\subsection{Calibration and Priors}

We calibrate a number of parameters. In particular, the discount factor $\beta$ is fixed at 0.9975 , corresponding to a $2.6 \%$ annual real interest rate at the prior mean. The steady-state depreciation rate $\delta$ is 0.025 , corresponding to a $10 \%$ depreciation rate per year. The elasticity of the demand for goods is set at 6 , which implies a $20 \%$ net price markup in steady state. We set the government spending-to-GDP ratio at $20 \%$, in line with its sample average.

Table 1 reports the prior distributions for the structural parameters of the model and the exogenous processes that drive the dynamics of the economy, which are set in accordance with Smets and Wouters (2007). The only differences relate to the Taylor rule coefficient associated with the response of the monetary authority to changes in the inflation rate $\left(\phi_{\pi}\right)$ and the fraction of ROT consumers $(\theta)$ which is absent in the RANK model of Smets and Wouters (2007). For $\phi_{\pi}$, Smets and Wouters (2007) specify a normal distribution truncated at 1 , centered at 1.50 and with standard deviation 0.25 and impose determinacy. Instead, here, we want to deal with the possibility of indeterminacy. Figure 1 shows the determinacy/indeterminacy regions as $\phi_{\pi}$ and $\theta$ vary. For low values of the fraction of ROT agents, the model behaves like a standard NK model, so that it admits a unique stable rational expectations equilibrium when the Taylor principle is satisfied, i.e., $\phi_{\pi}>1$. However, as it is well known from the literature, when $\theta$ is sufficiently high the result flips, so that the model needs a passive monetary policy, i.e., $\phi_{\pi}<1$, for determinacy to arise. Bilbiie (2008) calls this possibility the inverted-aggregatedemand-logic (IADL). The threshold value for $\theta$ that makes the model enter the IADL region of the parameter space depends on the properties of the model and on parameter calibration. While Bilbiie (2008) shows that in standard three equation NK model with ROT agents this threshold value for $\theta$ can be relatively low, Colciago (2011) shows that nominal wage rigidity increases the threshold value substantially (see also Ascari et al., 2017). ${ }^{4}$ In our mediumscale model, with parameters calibrated at their prior means, this threshold value in Figure 1 is around 0.6. Moreover, other possibilities arise in a medium-scale model, because some parameter combinations yield instability and some other a degree of indeterminacy greater than one. The next Section explains how we deal with the determinacy/indeterminacy issue

\footnotetext{
${ }^{4}$ Few papers analyse determinacy region in a medium-scale model with ROT. Motta and Tirelli (2012, 2014) highlight the role of the interaction between the fraction of ROT and the degree of habits in consumption. Neither paper includes capital and the related frictions. Albonico et al. (2019) show the results for the determinacy regions of a medium-scale model with respect to both the degree of habits and its specification.
} 
in the estimation, following Bianchi and Nicolò (2019). Regarding priors, we consider a prior which assigns roughly equal probability of observing indeterminacy as well as a unique solution. In particular, for $\phi_{\pi}$ we set a flatter normal prior distribution centered at 1 and with standard deviation 0.35 following Nicolò (2020). The fraction of ROT $\theta$ is assumed to follow a Beta distribution with mean 0.3 and standard deviation 0.1, in line with Bilbiie and Straub (2013).

\subsection{Methodology}

Bianchi and Nicolò (2019) develop a new method to solve and estimate linear rational expectations (LRE) models that accommodates both determinacy and indeterminacy. Their characterization of indeterminate equilibria is equivalent to Lubik and Schorfheide (2003, 2004) and Farmer, Khramov and Nicolò (2015). We closely follow Bianchi and Nicolò (2019) and in the following briefly sketch their methodology while referring the readers to their paper for detailed exposition. The LRE model can be compactly written in the canonical form as

$$
\Gamma_{0}(\Theta) s_{t}=\Gamma_{1}(\Theta) s_{t-1}+\Psi(\Theta) \varepsilon_{t}+\Pi(\Theta) \eta_{t}
$$

where $s_{t}$ is the vector of endogenous variables, $\Theta$ is the vector of model parameters, $\varepsilon_{t}$ is the vector of fundamental shocks, and $\eta_{t}$ are one-step ahead forecast errors for the expectational variables. Bianchi and Nicolò (2019) propose to augment the original model by appending an independent process, which could be either stable or unstable. First, for our medium-scale ROT model with priors set as above, the occurrence of indeterminacy of degree two (or higher) is a-priori very low and so in what follows we focus on one degree of indeterminacy. Second, the priors are such that there is roughly a 50-50 prior probability of determinacy and one degree of indeterminacy. Following Bianchi and Nicolò (2019), we append the following autoregressive process to the original LRE model

$$
\omega_{t}=\varphi^{*} \omega_{t-1}+\nu_{t}-\eta_{f, t}
$$

where $\nu_{t}$ is the sunspot shock and $\eta_{f, t}$ can be any element of the forecast error vector $\eta_{t}$. As proven by Bianchi and Nicolò (2019), it is without loss of generality that we include the forecast error associated with the inflation rate $\eta_{\pi, t}=\pi_{t}-E_{t-1}\left(\pi_{t}\right)$ as $\eta_{f, t}$ in the augmented representa- 
tion. The key insight consists of choosing this auxiliary process in a way to deliver the 'correct' solution. When the original model is determinate, the auxiliary process must be stationary so that the augmented representation also satisfies the Blanchard-Kahn condition. Accordingly, we set $\varphi^{*}$ such that its absolute value is inside the unit circle. Then the autoregressive process for $\omega_{t}$ does not affect the solution for the endogenous variables $s_{t}$. On the other hand, under indeterminacy, the additional process should be explosive so that the Blanchard-Kahn condition is satisfied for the augmented system, though it is not for the original model. Hence, the absolute value of $\varphi^{*}$ is set outside the unit circle. Under indeterminacy, we estimate the standard deviation of the sunspot shock, $\sigma_{\nu}$, and so we specify a uniform distribution over the interval $[0,1]$ following Nicolò $(2020)$. In addition, the newly defined sunspot shock, $\nu_{t}$, is potentially related to the structural shocks of the model. Nicolò (2020) finds that the correlation between this newly defined sunspot shock and the price markup shock is the only one statistically different from zero, implying that the price markup shock has a contemporaneous effect on inflation through this channel. Hence in what follows, we report estimation results corresponding to the correlations with the remaining shocks set to zero. ${ }^{5}$ For the correlation between the sunspot shock and the price markup shock, we set a uniform prior distribution over the interval $[-1,1]$ as in Nicolò (2020).

We use Bayesian techniques to estimate the model parameters and to test for (in)determinacy using posterior model probabilities. First, we find the mode of the posterior distribution by maximizing the log posterior function, which combines the prior information on the parameters with the likelihood of the data. In a second step, the Metropolis-Hastings algorithm is used to simulate the posterior distribution and to evaluate the marginal likelihood of the model. ${ }^{6}$

\section{Results}

We estimate both our baseline model and a model without ROT (where $\theta=0$ ) for the preVolcker (55:Q4-79:Q2) and the Great Moderation (84:Q1-07:Q3) periods separately. ${ }^{7}$ Table

\footnotetext{
${ }^{5}$ We also confirm that this is actually favoured by the data.

${ }^{6}$ All estimations are done using Dynare (https://www.dynare.org/wp-repo/dynarewp001.pdf). The posterior distributions are based on 500,000 draws, with the first 100,000 draws being discarded as burn-in draws. The average acceptance rate is around $25-30 \%$.

${ }^{7}$ We exclude the years of the Volcker disinflation and the end of the second subsample is marked by the onset of the Great Recession.
} 
2 shows the log-data densities of the four possibilities (determinacy vs. indeterminacy, ROT vs. RANK) for both subsamples. Comparing the log-likelihoods, both models (ROT and RANK) point definitely toward indeterminacy in the first subsample and determinacy in the second subsample. The probability of indeterminacy and determinacy in the two subsamples, respectively, are calculated as in Lubik and Schorfheide (2004) and are equal to one in both cases.

Then, let us focus on the first subsample under indeterminacy. The ROT model is marginally preferred to the RANK model. The Bayes factor comparing the two alternative models is 1.7, which according to the classification in Kass and Raftery (1995) is "not worth more than a bare mention" as evidence against the RANK model. ${ }^{8}$ Indeed, the two models are very close, so that our estimates deliver two main results.

First, consistent with most of the results in the literature (e.g., Lubik and Schorfheide, 2004, or more recently Nicolò, 2020), the RANK model in the first sub-sample yields indeterminacy, because of a passive monetary policy rule (the estimated posterior mean for $\phi_{\pi}$ is 0.798 , see Table 1). However, contrary to the evidence in Bilbiie and Straub (2013), this is also the case for the ROT model. The estimated posterior mean for the fraction of ROT, $\theta$, is low, equal to 0.219, far below the threshold value for the IADL region in our model (recall the discussion in Section 3.2 and Figure 1). Figure 2 shows that data are informative for the posterior distribution for $\theta$. Bilbiie and Straub (2013) found that the data preferred determinacy when estimating a small-scale ROT model for the pre-Volcker period, as a result of passive monetary policy and a high fraction of ROT (their posterior mean for $\theta$ is 0.5 ), that is, the model being in the IADL region of the parameter space. According to our medium-scale model, instead, the ROT model delivers indeterminacy, exactly for the same reason as the RANK model: a passive monetary policy (the estimated posterior mean for $\phi_{\pi}$ is 0.796 , see Table 1 ). The estimated ROT fraction is too low to put the model in the IADL region.

Second, the estimated ROT fraction is actually so low that the two models are extremely similar, delivering almost identical estimated posterior means of all the parameters, variance and historical decompositions, and impulse response functions to shocks. Table 1 shows the posterior means for all the parameters; there are barely any differences across the two models

\footnotetext{
${ }^{8}$ We report the Bayes Factor as suggested in Kass and Raftery (1995), calculated as 2(log-data density H1 - log-data density H0), where the null hypothesis (H0) is always the less preferred model (while the alternative hypothesis, H1, is the preferred one). Hence, we weight evidence against the null hypothesis.
} 
and the estimates are consistent with the standard value in the RANK-DSGE literature. Table 3 presents the variance decompositions for the pre-Volcker period. For both models, output and consumption volatility is mainly determined by the technology and the risk-premium shocks (the later being relatively more important for consumption). Government spending shock is also important for output fluctuations. In both models, inflation volatility is mainly driven by the wage markup, the technology and the price markup shocks, but also by the sunspot shock. So inflation dynamics was driven by self-fulfilling expectations both for the RANK and the ROT model. This is confirmed by the historical decomposition of inflation and the output gap, shown in Figures 3 - 6. The narrative about the main drivers of U.S. business cycle fluctuations that comes out from the estimated DSGE model is the same in both models, and corroborate the results in Nicolò (2020). In the Great Inflation period of the '70s, the dynamics of the output gap is mainly driven by risk-premium shocks, which generate 'stagflation' dynamics under indeterminacy. A positive risk-premium shock has a contractionary effect on the economy, but because of passive monetary policy agents form self-fulfilling inflationary expectations (see the impulse responses in Figure A.4 in the Appendix). In the same period, high inflation is caused by technology shocks, demand shocks and the sunspot shock. Passive monetary policy alters the dynamics of inflation in response to shocks, particularly to technology, risk premium and monetary policy shocks. The presence of ROT consumers does not alter this interpretation of U.S. business cycle fluctuations during this subsample, because their fraction is too low. The impulse response functions to the different shocks almost overlap for the two models (indicated as ROT-IND and RANK-IND in the Figures) with two expected exceptions: the responses of aggregate consumption to the government spending shock and to the investment shock. ${ }^{9}$ Figure 7 shows that the positive reaction of output to a government spending shock induces higher consumption of the ROT consumers that only partially compensate the decrease in consumption of optimizing consumers, who adhere to standard Ricardian equivalence dynamics. As a result, aggregate consumption decreases much less in the ROT-IND model than in the RANK-IND one. Similarly, Figure 8 shows that in response to the investment shock, the increase in income pushes up the consumption of ROT consumers, while optimizing consumers decrease their consumption to finance the increase in investment. As a result, aggregate consumption decreases

\footnotetext{
${ }^{9}$ Hence, in the main text we just include the impulse response functions to these two shocks, while the others are confined to the Appendix.
} 
slightly on impact, but then it increases in the ROT-IND model, contrary to the RANK-IND one. However, these differences are quantitatively negligible regarding the narrative of U.S. business cycle fluctuations according to the two models. The historical decomposition figures demonstrate that these two shocks are not quantitatively important drivers of consumption fluctuations. The variance decompositions in Table 3 are also unaffected. ${ }^{10}$

To sum up, the estimations of the two empirically rich models in the pre-Volcker subsample yield two main results that contrast with the ones in Bilbiie and Straub (2013), who estimate a small-scale model. First, a model with ROT consumers delivers indeterminacy due to passive monetary policy, just like a standard RANK model. Second, the estimate of the fraction of ROT consumers is so low that the RANK and the ROT models deliver almost exactly the same dynamics and interpretation of aggregate U.S. business cycle fluctuations.

Therefore, the presence of ROT consumers is not substantive to explain these fluctuations. Indeed, the difference in the log-data densities between the RANK-IND and the ROT-IND models are negligible. The next Section presents further robustness checks on the two main results of our paper.

The results for the second subsample are less surprising and in line with the existing literature. Both the RANK and the ROT model point towards determinacy and active monetary policy (see Table 2). The posterior mean for $\theta$, as seen in Table 4 , is very low (0.1), such that the two models are even more similar. Again, the estimated posterior means of all the other parameters of the model (see Table 4), the variance (see Table 5) and historical decompositions, and the impulse response functions are very similar across the two specifications, and they are in accordance with the results in Nicolò (2020). The Bayes factor (equal to 11) favours the RANK model 'very strongly', according to Kass and Raftery's (2015) classification. In accordance with the literature (Stock and Watson, 2003; Primiceri, 2005; Sims and Zha, 2006; Justiniano and Primiceri, 2008), the standard deviations of the fundamental shocks are substantially lower in this Great Moderation subsample, pointing to a change in both the shock volatilities and the conduct of monetary policy as the explanation for the conquest of American inflation.

\footnotetext{
${ }^{10}$ If anything, somewhat surprising, the fraction of the (forecast error) variance of consumption explained by these two shocks is higher in the RANK-IND model than in the ROT-IND one. While substantially so in percentage terms, the numbers are still miniscule.
} 


\subsection{Robustness: pre-Volcker sample}

Our main result concerns the irrelevance of ROT consumers for aggregate business cycle fluctuations in U.S. data. Given previous results in the literature, this is surprising for the pre-Volcker sample in particular. In this Section, we check the robustness of this result for the pre-Volcker sample with respect to changes to: (i) the prior for the fraction of ROT consumers, $\theta$; (ii) the specification of the Taylor rule; (iii) the subsample splits.

\subsubsection{Prior for $\theta$}

Our baseline prior for $\theta$ is in line with Bilbiie and Straub (2013). To give a fair chance to higher values for $\theta$, we re-estimate the model for the pre-Volcker period with a uniform prior $(0,1)$ for $\theta$. In this case, results are sensitive to the initial values, i.e. they depend on the region of the parameter space the estimations are launched in (as shown in Table A.1 in the Appendix). ${ }^{11}$ Starting from a parameter configuration from the usual determinacy region (standard aggregate demand logic, SADL, in Bilbiie's (2008) terminology), we find the same results as above, and the data strongly favour an indeterminate model. However, when we initialize the estimation algorithm in the IADL region, we do find results consistent with Bilbiie and Straub (2013). That is, we find determinacy due to a passive monetary policy (posterior mean of $\phi_{\pi}=0.50$ ) and a high value of ROT consumers (posterior mean of $\theta=0.65$ ) and, hence, the parameter estimates put the model in the IADL region. The log-data density, however, notably drops to $(-702.59)$, while it is equal to $(-609.66)$ for the indeterminate model estimated when the algorithm is initialized in the SADL region. The Bayes factor comparing these two log-data densities is as large as 185.9 signalling a very strong evidence against the determinate model with a high value of $\theta$.

\subsubsection{Forward-looking Taylor rule}

We run a robustness check assuming a forward-looking Taylor rule where the interest rate reacts to expected inflation as opposed to contemporaneous inflation as in our baseline model. Bilbiie (2008) shows that the 'inverted Taylor principle' holds in the IADL case in his small-scale NK model for a smaller fraction of ROT consumers with a forward-looking Taylor rule compared to

\footnotetext{
${ }^{11}$ This signals a problem of the estimation algorithm in allowing the crossing of the determinacy boundaries. Bianchi and Nicolò (2019) thoroughly discuss this problem.
} 
a contemporaneous Taylor rule. In addition, Bilbiie and Straub (2013) use a forward-looking Taylor rule whereby the monetary authority responds to expected inflation. First, we find that the determinacy-indeterminacy boundary with a forward-looking rule in our medium-scale model is the same as in Figure 1. Second, Table A.2 in the Appendix shows that the estimation results are very similar to our baseline results with contemporaneous inflation in the Taylor rule. $^{12}$

\subsubsection{Subsamples}

Table 6 displays the results of different experiments with four different subsamples for the Great Inflation years. The first two correspond to the two subsamples in Nicolò (2020), who argues that it is important to split the original sample in pre and post 1970, because the '70s are characterized by slower productivity growth, resulting into a distinct balanced growth path. Not surprisingly, our results are in line with Nicolò (2020) and the data favours the indeterminate model in both subsamples. Moreover, comparing the log-data densities, we show that there is 'positive' evidence against the ROT model compared to the RANK one. Hence, considering this split of our original pre-Volcker sample would reinforce our argument of rejecting the usefulness of a model with ROT consumers to fit the U.S. business cycle.

The third subsample (60:Q1-79:Q2) is the sample used by Lubik and Schorfheide (2004) and also by Bilbiie and Straub (2013). In this case, we find results similar to our baseline, so that the data favours the indeterminate model with basically no difference in terms of fit between the ROT and the RANK model. Hence, the fact that our results differ from the ones in Bilbiie and Straub (2013) is not due to us employing a different sample for the pre-Volcker period.

Finally, we experiment also with 66:Q1-79:Q2 which is the sample used in their seminal paper by Smets and Wouters (2007). To our surprise, here the results differ and it is worth spending few words on this fact because it might have been overlooked by the literature. Our results are consistent with Smets and Wouters (2007) because the data favour a determinate model for this particular subsample. Determinacy follows from the estimate of an active monetary policy and a small fraction of ROT consumers. In Kass and Raftery's (1995) terminology, there is positive evidence against indeterminacy. This is true for both the ROT and the RANK model,

\footnotetext{
${ }^{12}$ This is also true for most parameter estimates. For this exercise, we used a Uniform $(0,1)$ prior for $\theta$, while all the other priors are same as before.
} 
again signalling that the two models are empirically indistinguishable, despite the log-data density being marginally larger for the ROT model. Hence, whether or not the estimation finds indeterminacy in the pre-Volcker sample seems to be sensitive to the choice of the dates. We conjecture that the reason why the 66:Q1-79:Q2 sample yields determinacy is because of the increase in the real interest rate in the last years of ' 60 s that pushes the estimation towards an active monetary policy. The determinacy result seems to be confined to this particular sample period, so this could be just a minor point. However, given that papers in the literature might choose this sample period to compare their results with Smets and Wouters (2007), we think its important to point out that choosing this particular sample has an impact on the long standing debate about bad vs. good monetary policy in the pre-Volcker period.

\section{Conclusion}

We estimate a medium-scale model with ROT consumers over two different subsamples (the pre-Volcker and the Great Moderation periods), while allowing and testing for (in)determinacy, and compare our results with the standard RANK specification. Our main finding is that including ROT in a RANK model is irrelevant to explain U.S. aggregate business cycle fluctuations. The reason being that the ROT model preferred by the data has a very low fraction of ROT consumers, that only marginally affects the dynamics of the model relative to a RANK specification. The two models are basically empirically equivalent. In both subsamples, the RANK and ROT models yield almost the same impulse response functions, variance and historical decompositions, such that they share the same narrative of U.S. business cycle fluctuations

In line with Lubik and Schorfheide (2004) and Nicolò (2020), we find that passive monetary policy and self-fulfilling fluctuations characterize the pre-Volcker period for both the ROT and the RANK model. This contrasts with previous findings in the literature by Bilbiie and Straub (2013), who employ a small-scale model. In the pre-Volcker period the log-likelihoods of the ROT and the RANK model are very close, while in the second period the RANK model is preferred by the data.

Our main finding, that including ROT in a RANK model does not change the interpretation of aggregate U.S. business cycle fluctuations, does not mean that modelling ROT, or heterogeneous agents more generally, is not important to explain other dimensions of the data. 
However, in line with some others in the HANK literature (e.g., Bayer et al., 2020), it suggests that adding heterogeneity may not be substantive to explain aggregate fluctuations, at least for U.S. data.

\section{References}

[1] Albonico, A., Paccagnini, A., P. Tirelli, 2019, Limited Asset Market Participation and the Euro Area Crisis: an Empirical DSGE Model. Economic Inquiry, 57, 1302-1323.

[2] Ascari, G., A. Colciago, L. Rossi, 2017, Determinacy analysis in high order dynamic systems: The case of nominal rigidities and limited asset market participation, Economics Letters, vol. 159, 82-87.

[3] Bayer, C., B. Born, R. Luetticke, 2020, Shocks, Frictions, and Inequality in US Business Cycles, CEPR Discussion Paper 14364.

[4] Bianchi, F. and G. Nicolò, 2019. A Generalized Approach to Indeterminacy in Linear Rational Expectations Models, Finance and Economics Discussion Series 2019-033, Board of Governors of the Federal Reserve System (U.S.).

[5] Bilbiie, F.O., 2008. Limited asset market participation, monetary policy and (inverted) aggregate demand logic. Journal of Economic Theory 140, 162-196.

[6] Bilbiie, F.O., 2020. The New Keynesian cross. Journal of Monetary Economics 114, 90-108.

[7] Bilbiie, F.O. and R. Straub, 2012. Changes in the output Euler equation and asset markets participation, Journal of Economic Dynamics and Control, Elsevier, vol. 36(11), pages 1659-1672.

[8] Bilbiie, F.O. and R. Straub, 2013. Asset Market Participation, Monetary Policy Rules, and the Great Inflation, The Review of Economics and Statistics, MIT Press, vol. 95(2), pages 377-392, May.

[9] Brayton, F., T. Laubach, D. Reifschneider, 2014. The FRB/US Model: A Tool for Macroeconomic Policy Analysis, FEDS Notes. Washington: Board of Governors of the Federal Reserve System, April 03, 2014. https://doi.org/10.17016/2380-7172.0012 
[10] Calvo, G.A., 1983. Staggered prices in a utility-maximizing framework, Journal of Monetary Economics, Elsevier, vol. 12(3), pages 383-398, September.

[11] Christiano, L.J., M. Eichenbaum, C.L. Evans, 2005. Nominal Rigidities and the Dynamic Effects of a Shock to Monetary Policy, Journal of Political Economy, University of Chicago Press, vol. 113(1), pages 1-45, February.

[12] Coenen, G., R. Straub, M. Trabandt, 2012. Fiscal Policy and the Great Recession in the Euro Area, American Economic Review, Papers \& Proceedings, American Economic Association, vol. 102(3), pages 71-76, May.

[13] Colciago, A., 2011. Rule-of-thumb consumers meet sticky wages. Journal of Money, Credit and Banking 43, 325-353.

[14] Colciago, A., A. Samarina, J. de Haan, 2019. Central Bank Policies And Income And Wealth Inequality: A Survey, Journal of Economic Surveys, Wiley Blackwell, vol. 33(4), pages 1199-1231, September.

[15] Debortoli, D. and J. Galí, 2017. Monetary policy with heterogeneous agents: Insights from TANK models, Economics Working Papers 1686, Department of Economics and Business, Universitat Pompeu Fabra, revised Jun 2018.

[16] Farmer, R.E.A., V. Khramov, G. Nicolò, 2015. Solving and estimating indeterminate DSGE models, Journal of Economic Dynamics and Control, Elsevier, vol. 54(C), pages $17-36$.

[17] Forni, L., L. Monteforte, L. Sessa, 2009. The General Equilibrium Effects of Fiscal Policy: Estimates for the Euro Area, Journal of Public Economics, 93(3-4), 559-85.

[18] Galí, J., D. López-Salido, J. Vallés, 2007. Understanding the effects of government spending on consumption, Journal of the European Economic Association 5 (1), 227-270.

[19] Justiniano, A., and G.E. Primiceri. 2008. The Time-Varying Volatility of Macroeconomic Fluctuations. American Economic Review, 98 (3): 604-41.

[20] Justiniano, A., G. Primiceri, A. Tambalotti, 2010. Investment shocks and business cycles, Journal of Monetary Economics, Elsevier, vol. 57(2), pages 132-145, March. 
[21] Kaplan, G., B. Moll, G.L. Violante, 2018. Monetary Policy According to HANK, American Economic Review, 108 (3): 697-743.

[22] Kaplan, G., G.L. Violante, J. Weidner, 2014. The Wealthy Hand-to-Mouth, Brookings Papers on Economic Activity, Economic Studies Program, The Brookings Institution, vol. $45(1$ (Spring), pages 77-153.

[23] Kass, R. E. and A. E. Raftery. 1995. Bayes Factors, Journal of the American Statistical Association 90 (430): 773-95

[24] Kimball, M.S., 1995. The Quantitative Analytics of the Basic Neomonetarist Model, Journal of Money, Credit and Banking, Blackwell Publishing, vol. 27(4), pages 1241-77, November.

[25] Kollmann, R., B. Pataracchia, R. Raciborski, M. Ratto, W. Roeger, L. Vogel, 2016. The post-crisis slump in the Euro Area and the US: Evidence from an estimated three-region DSGE model, European Economic Review, Elsevier, vol. 88(C), pages 21-41.

[26] Lubik, T.A. and F. Schorfheide, 2004. Testing for Indeterminacy: An Application to U.S. Monetary Policy, American Economic Review, American Economic Association, vol. 94(1), pages 190-217, March.

[27] Lubik, T.A. and F. Schorfheide, 2003. Computing sunspot equilibria in linear rational expectations models, Journal of Economic Dynamics and Control, Elsevier, vol. 28(2), pages 273-285, November.

[28] Motta, G. and P. Tirelli, 2012, Optimal Simple Monetary and Fiscal Rules under Limited Asset Market Participation. Journal of Money, Credit and Banking, 44(7), 1351-74.

[29] Motta, G. and P. Tirelli, 2014. Limited Asset Market Participation, Income Inequality and Macroeconomic Volatility, Working Papers 65170975, Lancaster University Management School, Economics Department.

[30] Nicolò, G., 2020. Monetary Policy, Self-Fulfilling Expectations and the U.S. Business Cycle, Finance and Economics Discussion Series 2020-035, Board of Governors of the Federal Reserve System, https://doi.org/10.17016/FEDS.2020.035. 
[31] Primiceri, G. E., 2005. Time Varying Structural Vector Autoregressions and Monetary Policy, Review of Economic Studies, Oxford University Press, vol. 72(3), pages 821-852.

[32] Sims, C. A., and T. Zha, 2006. Were There Regime Switches in U.S. Monetary Policy?, American Economic Review, American Economic Association, vol. 96(1), pages 54-81, March.

[33] Slacalek, J., O. Tristani, G.L. Violante, 2020. Household balance sheet channels of monetary policy: A back of the envelope calculation for the euro area, Journal of Economic Dynamics and Control, Elsevier, vol. 115(C).

[34] Smets, F., R. Wouters , 2007. Shocks and Frictions in US Business Cycles: A Bayesian DSGE Approach, American Economic Review, American Economic Association, vol. 97(3), pp. 586-606.

[35] Stock, J. H., and M.W. Watson. 2003. Forecasting Output and Inflation: The Role of Asset Prices, Journal of Economic Literature, 41 (3): 788-829. 


\section{Tables and Figures}

Table 1. Parameter estimates for the sample 55-79

\begin{tabular}{|c|c|c|c|c|c|c|c|c|c|c|}
\hline & & \multicolumn{3}{|c|}{ Priors } & \multicolumn{3}{|c|}{ ROT ind } & \multicolumn{3}{|c|}{ RANK ind } \\
\hline & & shape & mean & st. dev. & post. mean & $90 \% \mathrm{HI}$ & interval & post. mean & $90 \% \mathrm{H}$ & interval \\
\hline TR response to inflation & $\phi_{\pi}$ & norm & 1 & 0.35 & 0.796 & 0.618 & 0.984 & 0.798 & 0.620 & 0.985 \\
\hline TR response to output & $\phi_{y}$ & norm & 0.12 & 0.05 & 0.152 & 0.086 & 0.219 & 0.142 & 0.073 & 0.206 \\
\hline TR response to output growth & $\phi_{g y}^{9}$ & norm & 0.12 & 0.05 & 0.184 & 0.136 & 0.230 & 0.179 & 0.129 & 0.227 \\
\hline TR interest rate smoothing & $\phi_{R}$ & beta & 0.75 & 0.1 & 0.840 & 0.767 & 0.917 & 0.833 & 0.756 & 0.912 \\
\hline inverse Frisch elasticity & $\phi_{l}$ & gamm & 2 & 0.75 & 1.393 & 0.610 & 2.145 & 1.410 & 0.623 & 2.207 \\
\hline habits & $b$ & beta & 0.7 & 0.1 & 0.487 & 0.373 & 0.601 & 0.537 & 0.427 & 0.646 \\
\hline investment adjustment costs & $\gamma_{I}$ & gamm & 4 & 1.5 & 4.563 & 2.496 & 6.476 & 4.896 & 3.023 & 6.868 \\
\hline Calvo price stickiness & $\xi_{p}$ & beta & 0.5 & 0.1 & 0.724 & 0.641 & 0.811 & 0.725 & 0.646 & 0.809 \\
\hline Calvo wage stickiness & $\xi_{w}$ & beta & 0.5 & 0.1 & 0.874 & 0.825 & 0.927 & 0.856 & 0.796 & 0.918 \\
\hline price indexation & $\chi_{p}$ & beta & 0.5 & 0.15 & 0.275 & 0.095 & 0.445 & 0.269 & 0.094 & 0.443 \\
\hline wage indexation & $\chi_{w}$ & beta & 0.5 & 0.15 & 0.373 & 0.178 & 0.552 & 0.374 & 0.185 & 0.565 \\
\hline capital utilization elasticity & $\sigma_{u}$ & beta & 0.5 & 0.15 & 0.397 & 0.205 & 0.584 & 0.455 & 0.265 & 0.647 \\
\hline ROT fraction & $\theta$ & beta & 0.3 & 0.1 & 0.219 & 0.131 & 0.309 & - & - & - \\
\hline intertemporal elasticity & $\sigma$ & norm & 1.5 & 0.37 & 1.309 & 0.996 & 1.645 & 1.358 & 1.036 & 1.690 \\
\hline capital share & $\alpha$ & norm & 0.3 & 0.05 & 0.192 & 0.159 & 0.224 & 0.191 & 0.157 & 0.223 \\
\hline ss growth & $g_{z}$ & norm & 0.4 & 0.1 & 0.292 & 0.192 & 0.394 & 0.283 & 0.189 & 0.378 \\
\hline ss hours & $\bar{h}$ & norm & 0 & 2 & -0.429 & -2.331 & 1.412 & -0.555 & -2.439 & 1.270 \\
\hline ss inflation & $\bar{\pi}$ & gamm & 0.62 & 0.1 & 0.616 & 0.455 & 0.779 & 0.614 & 0.449 & 0.770 \\
\hline \multicolumn{11}{|c|}{ Shocks persistences } \\
\hline risk premium & $\rho_{b}$ & beta & 0.5 & 0.2 & 0.755 & 0.617 & 0.901 & 0.743 & 0.610 & 0.884 \\
\hline investment & $\rho_{i}$ & beta & 0.5 & 0.2 & 0.629 & 0.488 & 0.769 & 0.680 & 0.545 & 0.824 \\
\hline monetary & $\rho_{r}$ & beta & 0.5 & 0.2 & 0.335 & 0.177 & 0.489 & 0.338 & 0.182 & 0.492 \\
\hline price markup & $\rho_{p}$ & beta & 0.5 & 0.2 & 0.350 & 0.039 & 0.675 & 0.364 & 0.048 & 0.692 \\
\hline wage markup & $\rho_{w}$ & beta & 0.5 & 0.2 & 0.837 & 0.677 & 0.985 & 0.829 & 0.641 & 0.989 \\
\hline government spending & $\rho_{g}$ & beta & 0.5 & 0.2 & 0.913 & 0.869 & 0.960 & 0.908 & 0.862 & 0.954 \\
\hline technology & $\rho_{a}$ & beta & 0.5 & 0.2 & 0.984 & 0.975 & 0.993 & 0.982 & 0.971 & 0.993 \\
\hline \multicolumn{11}{|c|}{ Shocks other parameters } \\
\hline MA component price markup & $\rho_{m a}^{p}$ & beta & 0.5 & 0.2 & 0.560 & 0.305 & 0.844 & 0.525 & 0.272 & 0.777 \\
\hline MA componentwage markup & $\rho_{m a}^{m a}$ & beta & 0.5 & 0.2 & 0.655 & 0.440 & 0.885 & 0.624 & 0.375 & 0.860 \\
\hline gov spending-tech correlation & $\rho_{g y}$ & norm & 0.5 & 0.25 & 0.590 & 0.473 & 0.706 & 0.602 & 0.484 & 0.716 \\
\hline \multicolumn{11}{|c|}{ Shocks standard deviations } \\
\hline risk premium & $\sigma_{b}$ & invg & 0.1 & 2 & 0.222 & 0.141 & 0.295 & 0.222 & 0.141 & 0.295 \\
\hline investment & $\sigma_{i}$ & invg & 0.1 & 2 & 0.441 & 0.324 & 0.548 & 0.441 & 0.324 & 0.548 \\
\hline monetary & $\sigma_{r}$ & invg & 0.1 & 2 & 0.177 & 0.153 & 0.201 & 0.177 & 0.153 & 0.201 \\
\hline price markup & $\sigma_{p}$ & invg & 0.1 & 2 & 0.372 & 0.308 & 0.434 & 0.372 & 0.308 & 0.434 \\
\hline wage markup & $\sigma_{w}$ & invg & 0.1 & 2 & 0.226 & 0.183 & 0.269 & 0.226 & 0.183 & 0.269 \\
\hline government spending & $\sigma_{g}$ & invg & 0.1 & 2 & 0.480 & 0.422 & 0.538 & 0.480 & 0.422 & 0.538 \\
\hline technology & $\sigma_{a}$ & invg & 0.1 & 2 & 0.711 & 0.622 & 0.799 & 0.711 & 0.622 & 0.799 \\
\hline sunspot & $\sigma_{\nu}$ & unif & 0.5 & 0.289 & 0.195 & 0.122 & 0.265 & 0.195 & 0.122 & 0.265 \\
\hline \multicolumn{11}{|c|}{ Shocks correlations } \\
\hline corr sunspot, price markup & $\rho_{\nu p}$ & unif & 0 & 0.577 & 0.821 & 0.648 & 1.000 & 0.821 & 0.648 & 1.000 \\
\hline
\end{tabular}


Table 2. Determinacy versus Indeterminacy

\begin{tabular}{ccccccc}
\hline \hline \multirow{2}{*}{ Sample } & Model & \multicolumn{2}{c}{ Log-data density } & \multicolumn{2}{c}{ Probability } & KR ratio \\
\cline { 3 - 6 } & Determinacy & Indeterminacy & Determinacy & Indeterminacy & 31.6 \\
\hline 1955Q4-1979Q2 & ROT & -624.85 & -609.07 & 0 & 1 & 18.5 \\
& RANK & -619.20 & -609.94 & 0 & 1 & 11.1 \\
KR ratio & & 11.3 & 1.7 & 1 & 0 & 11.0 \\
\hline 1984Q1-2007Q3 & ROT & -403.26 & -408.82 & 1 & 0 & \\
KR ratio & RANK & -397.69 & -403.17 & & & \\
\hline
\end{tabular}

Notes: The prior probability of determinacy is 0.51. ROT and RANK stand for Rule of Thumb and Representative Agent New Keynesian, respectively. Log marginal data densities are approximated by Geweke's (1999) harmonic mean estimator. The posterior probabilities are calculated based on the output of the Metropolis algorithm. KR stands for Kass and Raftery.

Table 3. Variance Decompositions (ROT-IND vs. RANK-IND), 1955Q4-1979Q2

\begin{tabular}{lcccccccc}
\hline & $\triangle c$ & $\triangle y$ & $\pi$ & $\triangle w$ & $\triangle i$ & $R$ & $\triangle c^{r t}$ & $\triangle c^{o}$ \\
\hline$R O T-I N D$ & & & & & & & & \\
$\varepsilon^{a}$ & 23.61 & 37.37 & 14.06 & 21.26 & 7.23 & 12.79 & 12.89 & 27.01 \\
$\varepsilon^{b}$ & 45.23 & 19.00 & 6.76 & 2.36 & 7.27 & 7.78 & 22.44 & 38.25 \\
$\varepsilon^{i}$ & 0.80 & 9.25 & 1.61 & 0.78 & 67.54 & 1.88 & 9.81 & 4.45 \\
$\varepsilon^{r}$ & 12.60 & 6.25 & 7.17 & 1.35 & 3.56 & 11.24 & 8.14 & 9.64 \\
$\varepsilon^{p}$ & 9.63 & 4.25 & 7.94 & 20.02 & 0.91 & 5.14 & 15.26 & 4.84 \\
$\varepsilon^{w}$ & 1.99 & 2.57 & 43.45 & 53.44 & 9.27 & 44.42 & 10.87 & 6.60 \\
$\varepsilon^{g}$ & 0.10 & 18.32 & 1.07 & 0.15 & 2.45 & 1.01 & 16.88 & 4.39 \\
$\varepsilon^{v}$ & 6.04 & 2.99 & 17.93 & 0.64 & 1.79 & 15.74 & 3.69 & 4.81 \\
\hline$R A N K-I N D$ & & & & & & & & \\
$\varepsilon^{a}$ & 27.46 & 43.48 & 15.77 & 22.19 & 9.14 & 13.65 & - & - \\
$\varepsilon^{b}$ & 44.91 & 19.78 & 8.41 & 3.36 & 8.64 & 9.46 & - & - \\
$\varepsilon^{i}$ & 1.74 & 7.54 & 0.89 & 0.45 & 68.53 & 0.95 & - & - \\
$\varepsilon^{r}$ & 9.73 & 5.34 & 7.19 & 1.45 & 3.90 & 12.92 & - & - \\
$\varepsilon^{p}$ & 5.71 & 3.25 & 12.72 & 20.87 & 1.52 & 8.74 & - & - \\
$\varepsilon^{w}$ & 5.19 & 3.15 & 38.95 & 51.13 & 6.18 & 40.30 & - & - \\
$\varepsilon^{g}$ & 1.50 & 15.49 & 0.53 & 0.04 & 0.67 & 0.47 & - & - \\
$\varepsilon^{v}$ & 3.76 & 1.98 & 15.55 & 0.52 & 1.41 & 13.52 & - & - \\
\hline
\end{tabular}


Table 4. Parameter estimates for the sample 84-07

\begin{tabular}{|c|c|c|c|c|c|c|c|}
\hline & & \multicolumn{3}{|c|}{ ROT det } & \multicolumn{3}{|c|}{ RANK det } \\
\hline & & post. mean & $90 \% \mathrm{HPD}$ & interval & post. mean & $90 \% \mathrm{HPD}$ & interval \\
\hline TR response to inflation & $\phi_{\pi}$ & 2.280 & 1.920 & 2.645 & 2.248 & 1.882 & 2.611 \\
\hline TR response to output & $\phi_{y}$ & 0.059 & 0.013 & 0.097 & 0.058 & 0.010 & 0.095 \\
\hline TR response to output growth & $\phi_{g y}$ & 0.167 & 0.117 & 0.219 & 0.169 & 0.119 & 0.219 \\
\hline TR interest rate smoothing & $\phi_{R}$ & 0.807 & 0.761 & 0.855 & 0.811 & 0.765 & 0.857 \\
\hline inverse Frisch elasticity & $\phi_{l}$ & 1.890 & 1.042 & 2.752 & 2.064 & 1.159 & 2.948 \\
\hline habits & $b$ & 0.421 & 0.309 & 0.527 & 0.439 & 0.331 & 0.539 \\
\hline investment adjustment costs & $\gamma_{I}$ & 5.614 & 3.197 & 7.971 & 5.983 & 3.497 & 8.405 \\
\hline Calvo price stickiness & $\xi_{p}$ & 0.801 & 0.733 & 0.874 & 0.803 & 0.737 & 0.871 \\
\hline Calvo wage stickiness & $\xi_{w}$ & 0.696 & 0.602 & 0.790 & 0.668 & 0.566 & 0.769 \\
\hline price indexation & $\chi_{p}$ & 0.471 & 0.257 & 0.682 & 0.473 & 0.254 & 0.684 \\
\hline wage indexation & $\chi_{w}$ & 0.523 & 0.282 & 0.760 & 0.513 & 0.271 & 0.761 \\
\hline capital utilization elasticity & $\sigma_{u}$ & 0.712 & 0.564 & 0.875 & 0.697 & 0.534 & 0.856 \\
\hline ROT fraction & $\theta$ & 0.105 & 0.052 & 0.157 & - & - & - \\
\hline intertemporal elasticity & $\sigma$ & 1.377 & 0.993 & 1.769 & 1.361 & 0.973 & 1.755 \\
\hline capital share & $\alpha$ & 0.177 & 0.140 & 0.215 & 0.179 & 0.143 & 0.216 \\
\hline ss growth & $g_{z}$ & 0.460 & 0.421 & 0.501 & 0.458 & 0.418 & 0.497 \\
\hline ss hours & $\bar{h}$ & -0.538 & -2.619 & 1.558 & -0.588 & -2.516 & 1.425 \\
\hline ss inflation & $\bar{\pi}$ & 0.655 & 0.524 & 0.785 & 0.660 & 0.530 & 0.788 \\
\hline \multicolumn{8}{|c|}{ Shocks persistences } \\
\hline risk premium & $\rho_{b}$ & 0.769 & 0.635 & 0.909 & 0.825 & 0.731 & 0.919 \\
\hline investment & $\rho_{i}$ & 0.683 & 0.558 & 0.814 & 0.698 & 0.567 & 0.827 \\
\hline monetary & $\rho_{r}$ & 0.361 & 0.206 & 0.517 & 0.354 & 0.201 & 0.511 \\
\hline price markup & $\rho_{p}$ & 0.883 & 0.799 & 0.970 & 0.882 & 0.795 & 0.977 \\
\hline wage markup & $\rho_{w}$ & 0.983 & 0.970 & 0.996 & 0.975 & 0.957 & 0.994 \\
\hline government spending & $\rho_{g}$ & 0.967 & 0.948 & 0.987 & 0.967 & 0.946 & 0.989 \\
\hline technology & $\rho_{a}$ & 0.944 & 0.911 & 0.978 & 0.935 & 0.897 & 0.972 \\
\hline \multicolumn{8}{|c|}{ Shocks other parameters } \\
\hline MA component price markup & $\rho_{m a}^{p}$ & 0.629 & 0.450 & 0.815 & 0.644 & 0.468 & 0.823 \\
\hline MA componentwage markup & $\rho_{m a}^{w}$ & 0.600 & 0.397 & 0.809 & 0.509 & 0.300 & 0.717 \\
\hline gov spending-tech correlation & $\rho_{g y}$ & 0.470 & 0.318 & 0.624 & 0.471 & 0.320 & 0.619 \\
\hline \multicolumn{8}{|c|}{ Shocks standard deviations } \\
\hline risk premium & $\sigma_{b}$ & 0.125 & 0.078 & 0.169 & 0.106 & 0.071 & 0.139 \\
\hline investment & $\sigma_{i}$ & 0.336 & 0.258 & 0.411 & 0.314 & 0.240 & 0.385 \\
\hline monetary & $\sigma_{r}$ & 0.121 & 0.104 & 0.138 & 0.120 & 0.103 & 0.137 \\
\hline price markup & $\sigma_{p}$ & 0.122 & 0.086 & 0.157 & 0.119 & 0.084 & 0.153 \\
\hline wage markup & $\sigma_{w}$ & 0.375 & 0.285 & 0.465 & 0.402 & 0.287 & 0.513 \\
\hline government spending & $\sigma_{g}$ & 0.379 & 0.334 & 0.425 & 0.380 & 0.334 & 0.427 \\
\hline technology & $\sigma_{a}$ & 0.406 & 0.356 & 0.455 & 0.406 & 0.356 & 0.454 \\
\hline
\end{tabular}


Table 5. Variance Decompositions (ROT-DET vs. RANK-DET), 1984Q1-2007Q3

\begin{tabular}{lcccccccc}
\hline & $\triangle c$ & $\triangle y$ & $\pi$ & $\triangle w$ & $\triangle i$ & $R$ & $\triangle c^{r t}$ & $\triangle c^{o}$ \\
\hline$R O T-D E T$ & & & & & & & & \\
$\varepsilon^{a}$ & 4.25 & 18.18 & 2.40 & 1.88 & 4.61 & 5.04 & 5.14 & 5.97 \\
$\varepsilon^{b}$ & 41.01 & 19.83 & 12.23 & 12.49 & 2.68 & 32.21 & 22.49 & 32.59 \\
$\varepsilon^{i}$ & 1.18 & 9.88 & 6.18 & 2.30 & 76.35 & 16.36 & 6.85 & 3.63 \\
$\varepsilon^{r}$ & 17.06 & 8.81 & 10.03 & 6.83 & 1.53 & 5.74 & 11.16 & 12.95 \\
$\varepsilon^{p}$ & 10.65 & 10.14 & 24.90 & 28.56 & 6.35 & 5.64 & 28.20 & 4.17 \\
$\varepsilon^{w}$ & 21.37 & 11.72 & 43.16 & 47.55 & 7.52 & 31.64 & 17.77 & 30.98 \\
$\varepsilon^{g}$ & 4.49 & 21.44 & 1.10 & 0.39 & 0.96 & 3.37 & 8.40 & 9.70 \\
\hline$R A N K-D E T$ & & & & & & & & \\
$\varepsilon^{a}$ & 6.43 & 20.84 & 2.26 & 1.88 & 4.13 & 4.61 & - & - \\
$\varepsilon^{b}$ & 36.34 & 18.76 & 18.44 & 15.40 & 3.28 & 44.89 & - & - \\
$\varepsilon^{i}$ & 2.69 & 9.18 & 4.59 & 1.75 & 73.98 & 11.39 & - & - \\
$\varepsilon^{r}$ & 15.54 & 8.40 & 9.39 & 7.65 & 1.69 & 6.52 & - & - \\
$\varepsilon^{p}$ & 7.46 & 9.51 & 27.64 & 25.86 & 9.05 & 7.17 & - & - \\
$\varepsilon^{w}$ & 24.66 & 12.53 & 36.91 & 47.13 & 7.41 & 23.25 & - & - \\
$\varepsilon^{g}$ & 6.87 & 20.78 & 0.76 & 0.33 & 0.47 & 2.17 & - & - \\
\hline
\end{tabular}

Table 6. Determinacy versus Indeterminacy - Sub-sample estimation

\begin{tabular}{|c|c|c|c|c|c|c|}
\hline \multirow[b]{2}{*}{ Sample } & \multirow[b]{2}{*}{ Model } & \multicolumn{2}{|c|}{ Log-data density } & \multicolumn{2}{|c|}{ Probability } & \multirow[t]{2}{*}{ KR ratio } \\
\hline & & Determinacy & Indeterminacy & Determinacy & Indeterminacy & \\
\hline \multirow[t]{2}{*}{ 1955Q4-1969Q4 } & $\mathrm{ROT}$ & -369.15 & -354.86 & 0 & 1 & 28.6 \\
\hline & RANK & -366.81 & -352.16 & 0 & 1 & 29.3 \\
\hline KR ratio & & 4.7 & 5.4 & & & \\
\hline \multirow[t]{2}{*}{ 1970Q1-1979Q2 } & ROT & -289.04 & -287.42 & 0.17 & 0.83 & 3.2 \\
\hline & RANK & -287.38 & -285.13 & 0.10 & 0.90 & 4.5 \\
\hline $\mathrm{KR}$ ratio & & 3.3 & 4.6 & & & \\
\hline \multirow{2}{*}{ 1960Q1-1979Q2 } & $\mathrm{ROT}$ & -507.91 & -503.89 & 0.02 & 0.98 & 8.0 \\
\hline & RANK & -519.03 & -505.23 & 0 & 1 & 27.6 \\
\hline KR ratio & & 22.2 & 2.7 & & & \\
\hline \multirow[t]{2}{*}{ 1966Q1-1979Q2 } & $\mathrm{ROT}$ & -368.85 & -371.30 & 0.92 & 0.08 & 4.9 \\
\hline & RANK & -370.24 & -371.87 & 0.84 & 0.16 & 3.3 \\
\hline KR ratio & & 2.8 & 1.1 & & & \\
\hline
\end{tabular}

Notes: The prior probability of determinacy is 0.51 . ROT and RANK stand for Rule of Thumb and Representative Agent New Keynesian, respectively. Log marginal data densities are approximated by Geweke's (1999) harmonic mean estimator. The posterior probabilities are calculated based on the output of the Metropolis algorithm. KR stands for Kass and Raftery. 


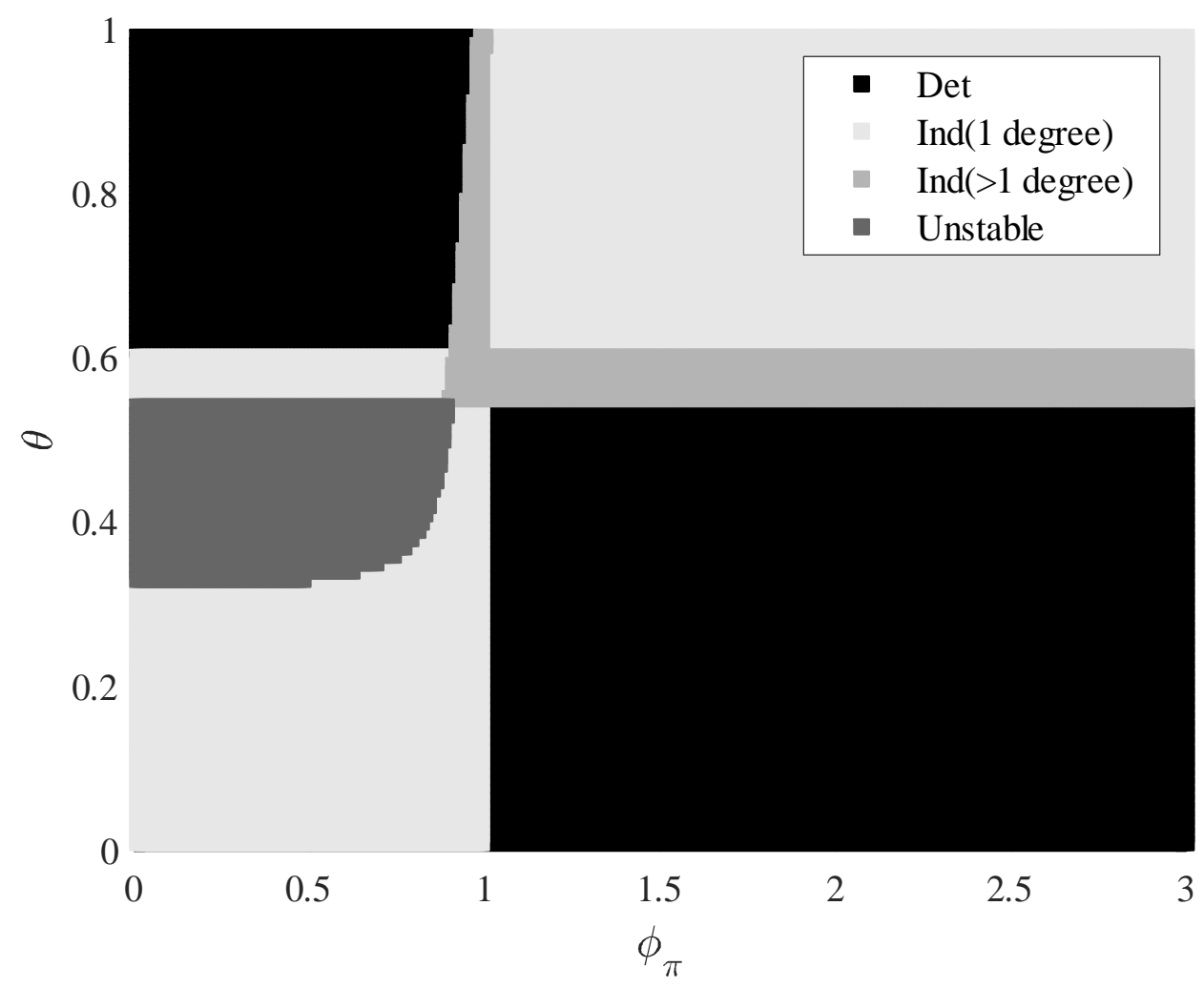

Figure 1: Determinacy region for $\phi_{\pi}$ against $\theta$; the remaining structural parameters of the model are set at the prior mean .

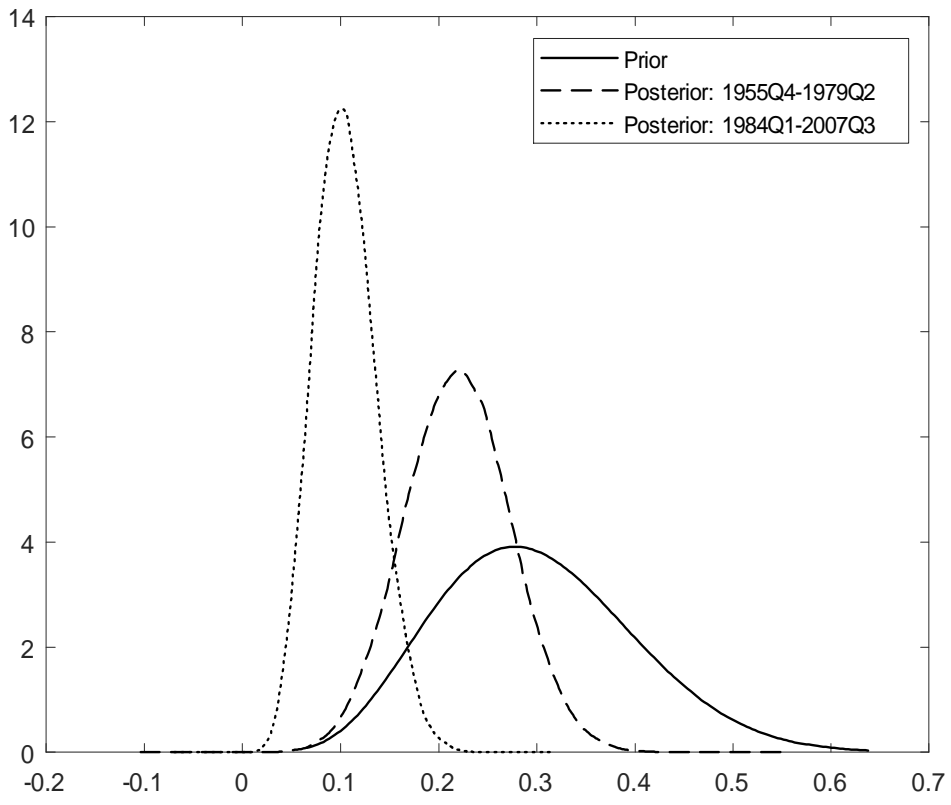

Figure 2: Prior-posterior plot for $\theta$ 


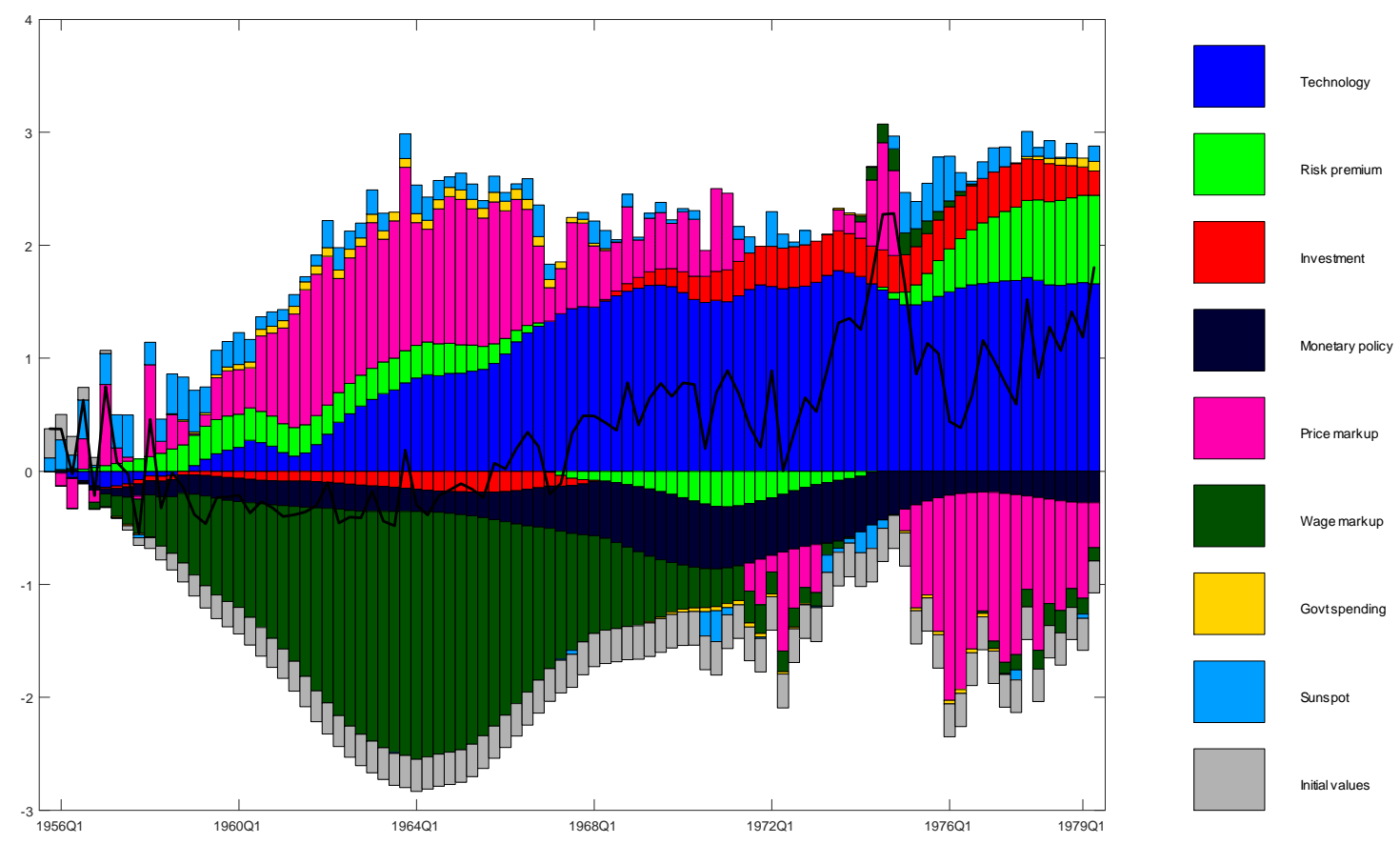

Figure 3: Historical Decomposition of Inflation from the ROT model under INDETERMINACY (Sample: 1955Q4-1979Q2).

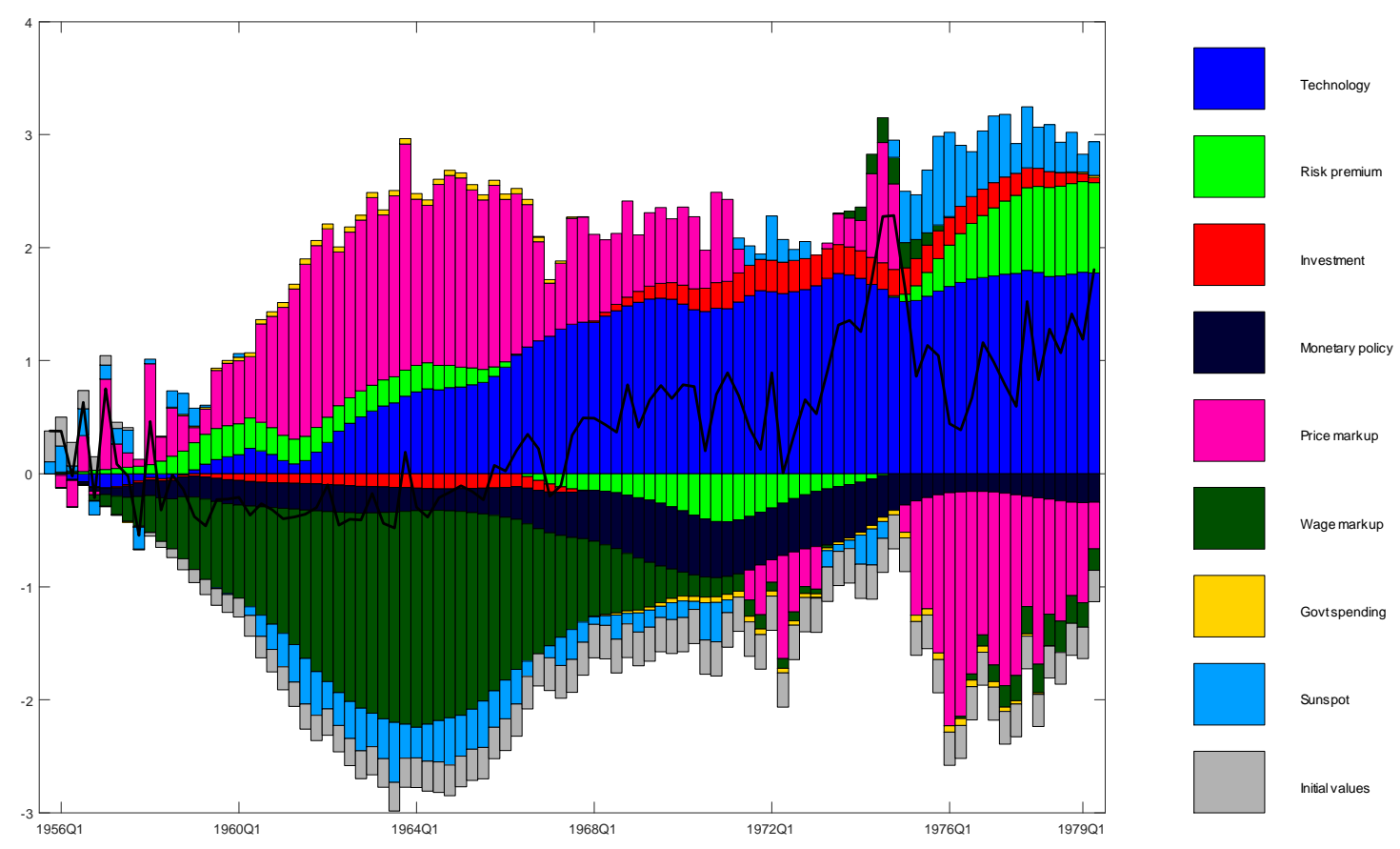

Figure 4: Historical Decomposition of Inflation from the RANK model under INDETERMINACY (Sample: 1955Q4-1979Q2). 


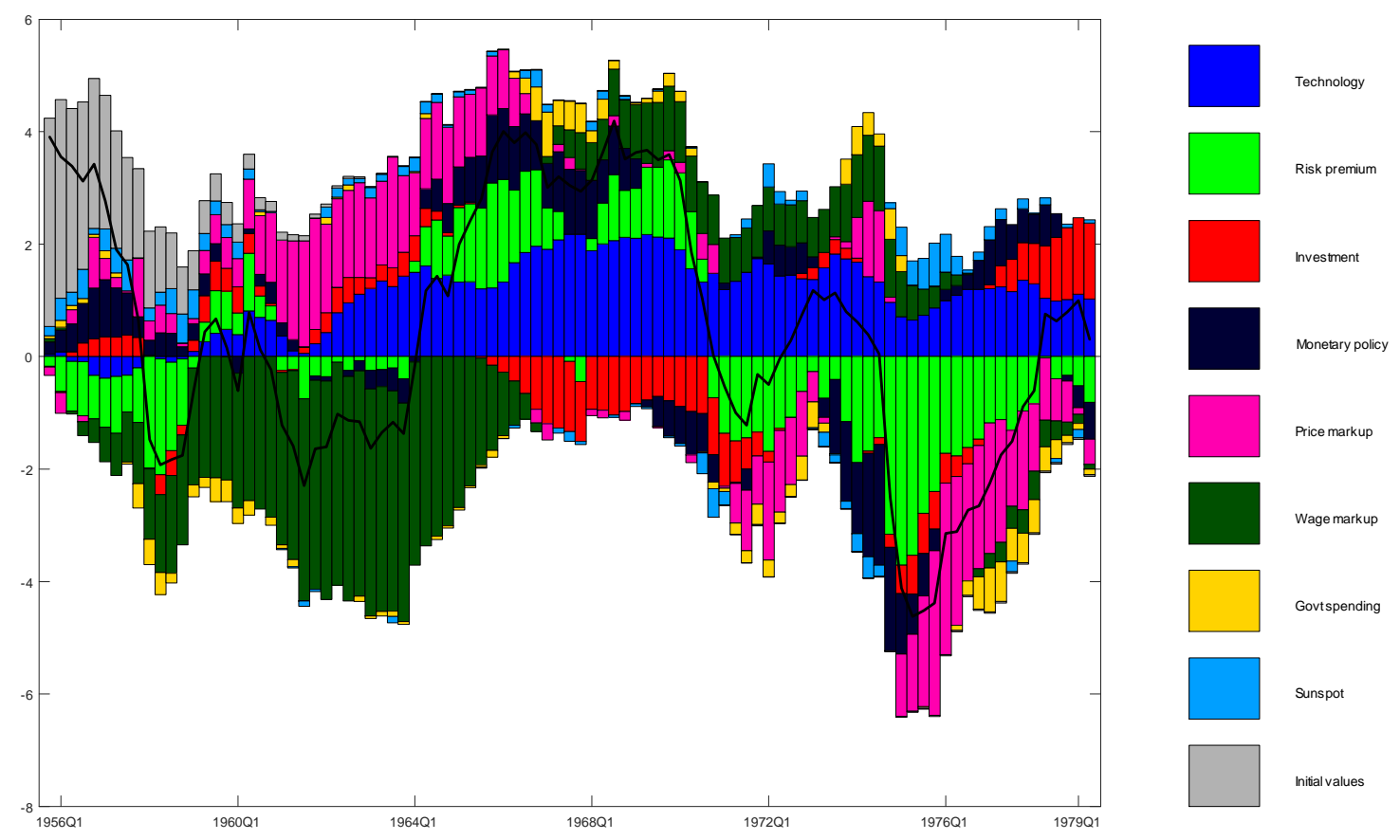

Figure 5: Historical Decomposition of the Output Gap from the ROT model under INDETERMINACY (Sample: 1955Q4-1979Q2).

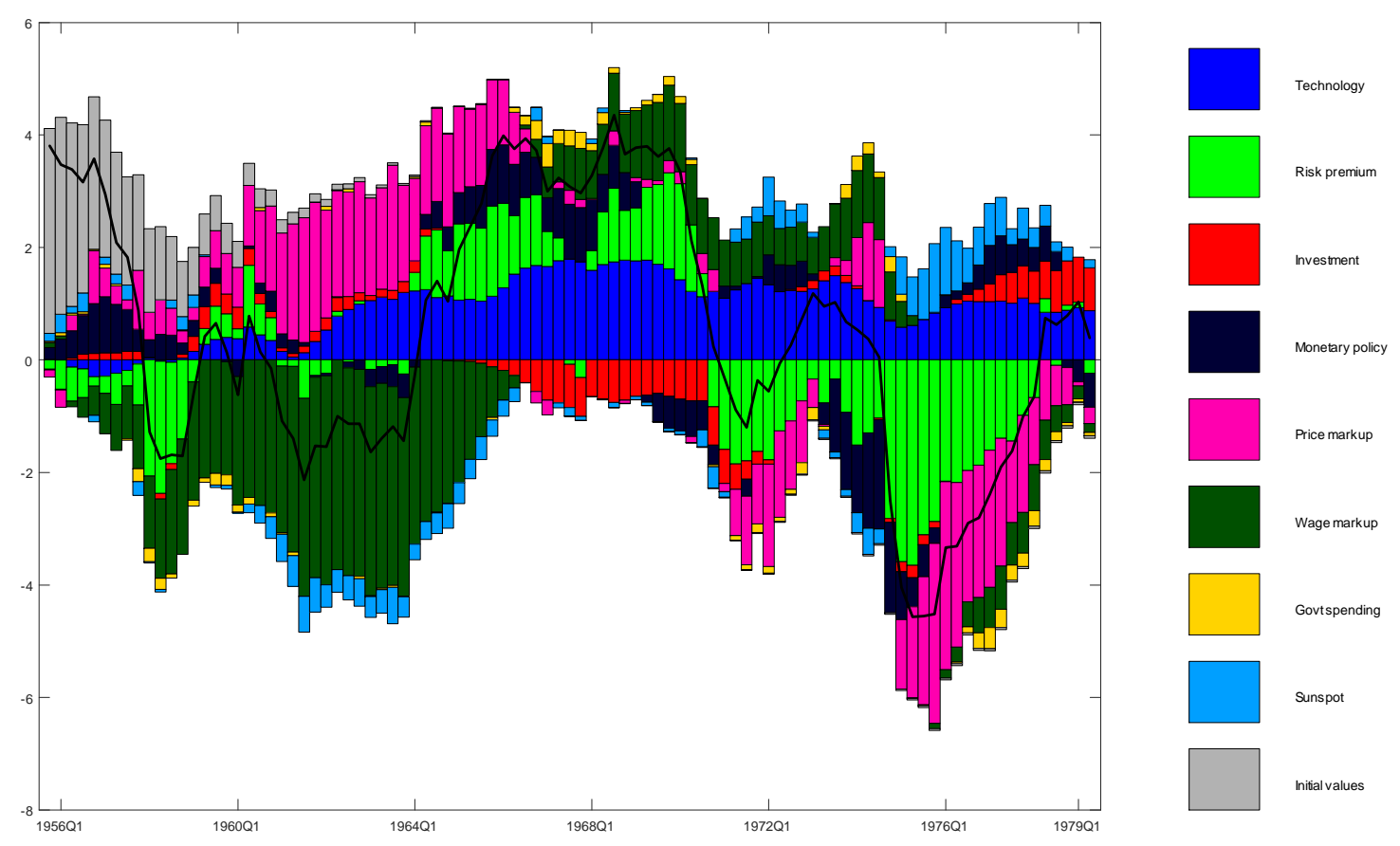

Figure 6: Historical Decomposition of the Output Gap from the RANK model under INDETERMINACY (Sample: 1955Q4-1979Q2). 

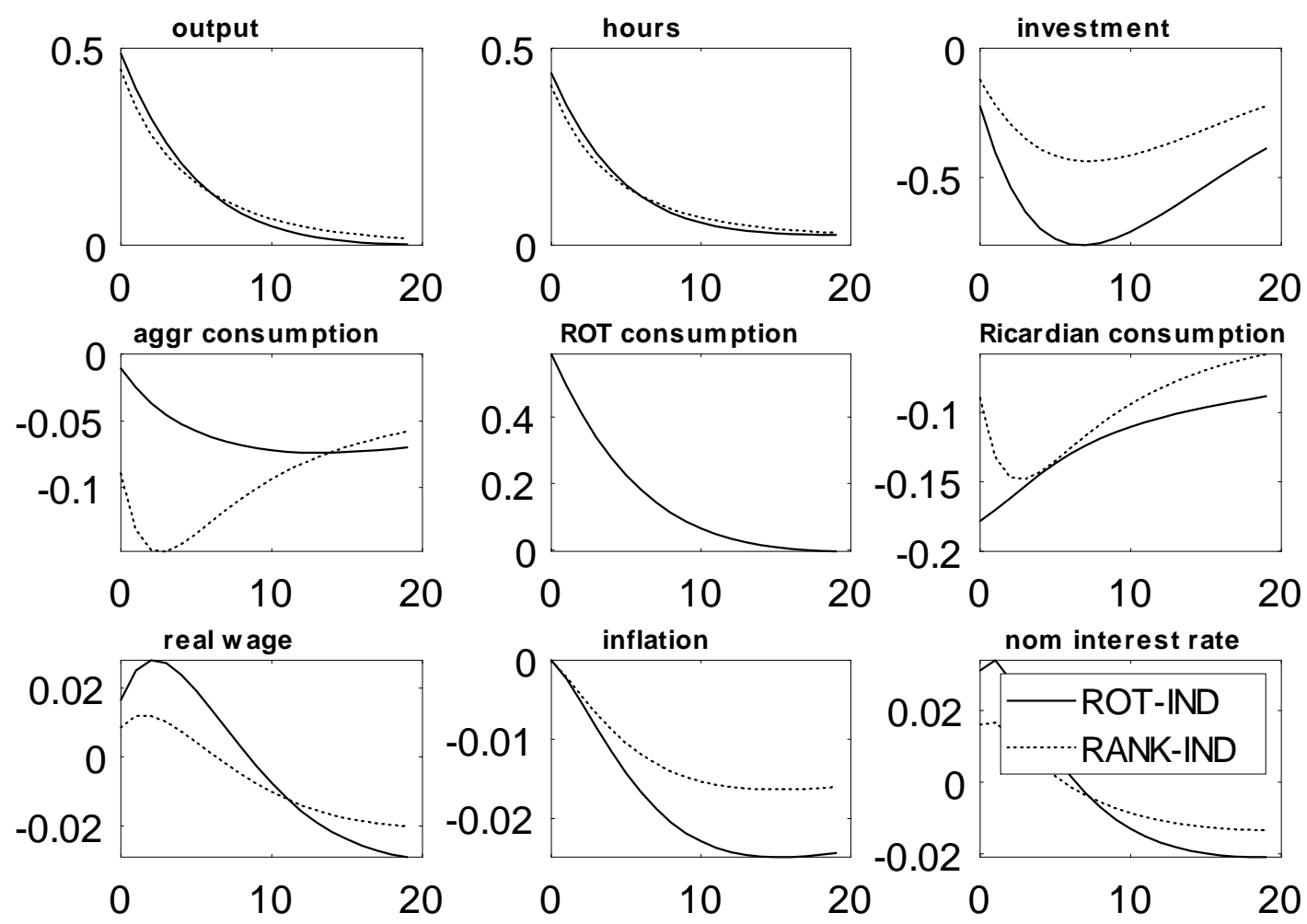

Figure 7: Impulse responses to a one standard deviation government spending shock (Sample: 1955Q4-1979Q2)
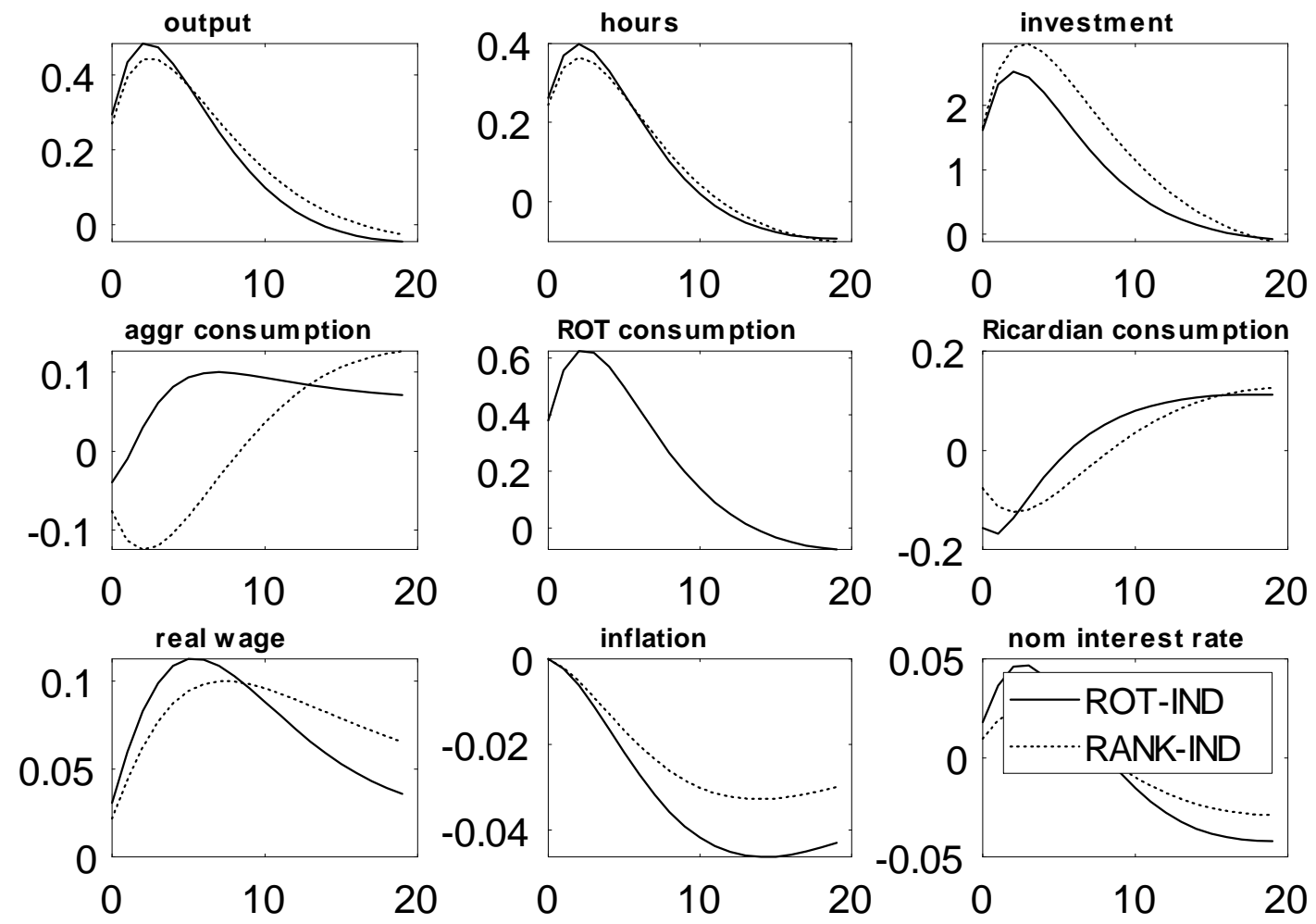

Figure 8: Impulse responses to a one standard deviation investment-specific shock (Sample: 1955Q4-1979Q2) 


\section{A Appendix}

\section{A.1 System of non-linear equations}

After deriving the first conditions of the model, we adjust variables to guarantes that the model has a balanced growth. Lower case letters stand for detrended variables, for example, $y_{t}=\frac{Y_{t}}{g_{z}^{t}}$, $w_{t}=\frac{W_{t}}{P_{t} g_{z}^{t}}, r_{t}^{k}=\frac{R_{t}^{k}}{P_{t}}, \lambda_{t}^{o}=\Lambda_{t}^{o} g_{z}^{t}$. Given that the model is then log-linearized, we omit price and wage dispersion variables. We add exogenous shock processes for the following variables: $\varepsilon_{t}^{a}$, $\varepsilon_{t}^{b}, \varepsilon_{t}^{i}, \varepsilon_{t}^{r}, \lambda_{t}^{p}, \lambda_{t}^{w}, g_{t}$. Lump-sum taxes are also modeled as exogenous shocks, which we are not estimating, thus they remain constant at their steady state. Given that the government budget constraint is balanced every period, we can omit this equation.

$$
\begin{aligned}
& \left(c_{t}^{o}-b c_{t-1}\right)^{-\sigma}=\lambda_{t}^{o} \\
& R_{t}=\pi_{t+1} g_{z} \frac{\lambda_{t}^{o}}{\beta \varepsilon_{t}^{b} \lambda_{t+1}^{o}} \\
& 1=Q_{t}^{o} \varepsilon_{t}^{i}\left\{1-\gamma_{I}\left(g_{z} \frac{i_{t}}{i_{t-1}}-g_{z}\right) g_{z} \frac{i_{t}}{i_{t-1}}-\frac{\gamma_{I}}{2}\left(g_{z} \frac{i_{t}}{i_{t-1}}-g_{z}\right)^{2}\right\} \\
& +g_{z} \frac{\lambda_{t+1}^{o}}{\lambda_{t}^{o}} Q_{t+1}^{o} \varepsilon_{t+1}^{i} \beta \gamma_{I}\left(g_{z} \frac{i_{t+1}}{i_{t}}-g_{z}\right)\left(\frac{i_{t+1}}{i_{t}}\right)^{2} \\
& \frac{1}{g_{z}} \frac{\lambda_{t+1}^{o}}{\lambda_{t}^{o}} \beta\left\{\left[r_{t+1}^{k} u_{t+1}-a\left(u_{t+1}\right)\right]+Q_{t+1}^{o}(1-\delta)\right\}=Q_{t}^{o} \\
& r_{t}^{k}=\gamma_{u 1}+\gamma_{u 2}\left(u_{t}-1\right) \\
& k_{t+1}=(1-\delta) \frac{k_{t}}{g_{z}}+\varepsilon_{t}^{i}\left[1-\frac{\gamma_{I}}{2}\left(g_{z} \frac{i_{t}}{i_{t-1}}-g_{z}\right)^{2}\right] i_{t} \\
& c_{t}^{r t}=w_{t}^{r t} h_{t} \\
& y_{t}=c_{t}+g_{t}+i_{t}+\frac{a\left(u_{t}\right) k_{t}}{g_{z}} \\
& c_{t}=\theta c_{t}^{r t}+(1-\theta) c_{t}^{o} \\
& 0=E_{t} \sum_{s=0}^{\infty}\left(\xi_{w} \beta\right)^{s}\left(\tilde{w}_{t}^{j}\right)^{-\frac{1+\lambda_{w}^{w}}{\lambda_{t}^{u}}}\left(\frac{\pi_{t, t+s-1}^{\chi_{w}} \bar{\pi}_{t, t+s}^{1-\chi_{w}}}{w_{t+s} \pi_{t, t+s}}\right)^{-\frac{1+\lambda_{t}^{w}}{\lambda_{t}^{t}}} h_{t+s}^{d} . \\
& \left\{\begin{array}{c}
\tilde{w}_{t}^{j} \frac{j_{t, t+s-1}^{\chi_{w}} \bar{\pi}_{t, t+s}^{1-\chi_{w}}}{\pi_{t, t+s}}\left(1-\frac{1+\lambda_{t}^{w}}{\lambda_{t}^{w}}\right)\left[(1-\theta)\left(c_{t+s}^{o}-b c_{t+s-1}\right)^{-\sigma}+\theta\left(c_{t+s}^{r t}-b c_{t+s-1}\right)^{-\sigma}\right] \\
+\frac{1+\lambda_{t}^{w}}{\lambda_{t}^{w}}\left[(1-\theta)\left(c_{t+s}^{o}-b c_{t+s-1}\right)^{-\sigma} M R S_{t+s}^{o}+\theta\left(c_{t+s}^{r t}-b c_{t+s-1}\right)^{-\sigma} M R S_{t+s}^{r t}\right]
\end{array}\right\}
\end{aligned}
$$




$$
\begin{gathered}
w_{t}=\left[\xi_{w}\left(\frac{\pi_{t-1}^{\chi_{w}} \bar{\pi}_{t}^{1-\chi_{w}}}{\pi_{t}} w_{t-1}\right)^{\frac{1}{\lambda_{t}^{w}}}+\left(1-\xi_{w}\right)\left(\tilde{w}_{t}\right)^{\frac{1}{\lambda_{t}^{w}}}\right]^{\lambda_{t}^{w}} \\
\frac{u_{t} k_{t}}{h_{t} g_{z}}=\frac{\alpha}{(1-\alpha)} \frac{w_{t}}{r_{t}^{k}} \\
m c_{t}=\alpha^{-\alpha}(1-\alpha)^{-(1-\alpha)}\left(\varepsilon_{t}^{a}\right)^{-1}\left(r_{t}^{k}\right)^{\alpha} w_{t}^{1-\alpha} \\
y_{t}=\varepsilon_{t}^{a}\left(u_{t} \frac{k_{t}}{g_{z}}\right)^{\alpha}\left(h_{t}^{d}\right)^{1-\alpha}-\Phi \\
E_{t} \sum_{s=0}^{\infty}\left(\xi_{p} \beta\right)^{s} \varepsilon_{t}^{b} \frac{\lambda_{t+s}^{o}}{\lambda_{t}^{o}} y_{t+s}^{z}\left[\tilde{p}_{t}^{z} \frac{\pi_{t, t+s-1}^{\chi_{p}} \bar{\pi}_{t, t+s}^{1-\chi_{p}}}{\pi_{t, t+s}}\left(1+\frac{1}{G^{\prime-1}\left(\omega_{t+s}\right)} \frac{G^{\prime}\left(x_{t+s}\right)}{G^{\prime \prime}\left(x_{t+s}\right)}\right)-m c_{t+s} \frac{1}{G^{\prime-1}\left(\omega_{t+s}\right)} \frac{G^{\prime}\left(x_{t+s}\right)}{G^{\prime \prime}\left(x_{t+s}\right)}\right]=0 \\
1=\left(1-\xi_{p}\right) \tilde{p}_{t}^{z} G^{\prime-1}\left(\tilde{p}_{t}^{z} \int_{0}^{1} G^{\prime}\left(\frac{y_{t}^{z}}{y_{t}}\right) \frac{y_{t}^{z}}{y_{t}} d z\right) \\
+\xi_{p} \pi_{t-1}^{\chi_{p}} \bar{\pi}_{t}^{1-\chi_{p}} \pi_{t}^{-1} G^{\prime-1}\left(\pi_{t-1}^{\chi_{p}} \bar{\pi}_{t}^{1-\chi_{p}} \pi_{t}^{-1} \int_{0}^{1} G^{\prime}\left(\frac{y_{t}^{z}}{y_{t}}\right) \frac{y_{t}^{z}}{y_{t}} d z\right) \\
h_{t}=h_{t}^{d}
\end{gathered}
$$

\section{A.2 System of log-linearized equations}

The above equations are log-linearized. We set the consumption ratio between the two groups $\left(c^{r t} / c^{o}\right)$ in steady state at 1 . Hatted variables are in log-deviation from their steady state. Government spending is expressed in deviation from steady state output, so that, $\tilde{g}_{t}=\frac{g_{t}-g}{y}$. We define $\varpi=\frac{\theta}{1-\theta}\left(\frac{\frac{c^{r t}}{c}-b}{\frac{c^{o}}{c}-b}\right)^{-\sigma}$ and $A=\frac{1}{\lambda^{p} \alpha^{p}+1}$, where $\alpha^{p}$ is elasticity of substitution between goods. It is implicit that the system below is completed with flexible prices and wages equilibrium conditions which are not reported here.

$$
\begin{gathered}
-\sigma \frac{1}{1-b \frac{c}{c^{o}}} \hat{c}_{t}^{o}+\sigma \frac{b}{\frac{c^{o}}{c}-b} \hat{c}_{t-1}=\hat{\lambda}_{t}^{o} \\
\hat{R}_{t}=-\hat{\varepsilon}_{t}^{b}+\hat{\pi}_{t+1}+\hat{\lambda}_{t}^{o}-\hat{\lambda}_{t+1}^{o} \\
\hat{\imath}_{t}=\frac{1}{\gamma_{I} g_{z}^{2}(1+\beta)}\left(\hat{Q}_{t}^{o}+\hat{\varepsilon}_{t}^{i}\right)+\frac{1}{1+\beta} \hat{\imath}_{t-1}+\frac{\beta}{1+\beta} \hat{\imath}_{t+1} \\
\hat{\lambda}_{t+1}^{o}-\hat{\lambda}_{t}^{o}+\frac{\beta}{g_{z}} r^{k} \hat{r}_{t+1}^{k}+\frac{\beta}{g_{z}}(1-\delta) \hat{Q}_{t+1}^{o}=\hat{Q}_{t}^{o} \\
\hat{r}_{t}^{k}=\frac{\gamma_{u 2}}{r^{k}} \hat{u}_{t} \\
\hat{k}_{t+1}=\frac{(1-\delta)}{g_{z}} \hat{k}_{t}+\frac{i}{k} \hat{\imath}_{t}+\frac{i}{k} \hat{\varepsilon}_{t}^{i}
\end{gathered}
$$




$$
\begin{aligned}
& \frac{c^{r t}}{c} \frac{c}{y} \hat{c}_{t}^{r t}=\frac{w h}{c} \frac{c}{y}\left(\hat{w}_{t}^{r t}+\hat{h}_{t}\right) \\
& 0=\frac{c}{y} \hat{c}_{t}+\tilde{g}_{t}+\frac{i}{y} \hat{\imath}_{t}-\hat{y}_{t}+\frac{\gamma_{u 1} k}{y g_{z}} \hat{u}_{t} \\
& \hat{c}_{t}=\theta \frac{c^{r t}}{c} \hat{c}_{t}^{r t}+(1-\theta) \frac{c^{o}}{c} \hat{c}_{t}^{o} \\
& \left(1+\beta \chi_{p}\right) \hat{\pi}_{t}=\chi_{p} \hat{\pi}_{t-1}+\beta \hat{\pi}_{t+1}+A \frac{\left(1-\beta \xi_{p}\right)\left(1-\xi_{p}\right)}{\xi_{p}}\left(\widehat{m c}_{t}+\hat{\lambda}_{t}^{p}\right) \\
& \hat{w}_{t}=-\frac{\left(1-\xi_{w}\right)\left(1-\xi_{w} \beta\right)}{(1+\beta) \xi_{w}} \hat{w}_{t}+\frac{\left(1-\xi_{w}\right)\left(1-\xi_{w} \beta\right)}{(1+\beta) \xi_{w}} \frac{\lambda^{w}}{1+\lambda^{w}} \hat{\lambda}_{t}^{w} \\
& +\frac{\left(1-\xi_{w}\right)\left(1-\xi_{w} \beta\right)}{(1+\beta) \xi_{w}} \frac{1}{1+\varpi}\left(\widehat{M R S}_{t}^{o}+\varpi \widehat{M R S}_{t}^{r t}\right) \\
& +\frac{\beta}{1+\beta} \hat{w}_{t+1}+\frac{1}{1+\beta} \hat{w}_{t-1}+\frac{\chi_{w}}{1+\beta} \hat{\pi}_{t-1}-\frac{\left(1+\beta \chi_{w}\right)}{1+\beta} \hat{\pi}_{t}+\frac{\beta}{1+\beta} \hat{\pi}_{t+1} \\
& \widehat{M R S}_{t}^{o}=\frac{\sigma}{1-b \frac{c}{c^{o}}} \hat{c}_{t}^{o}-\frac{b \sigma}{\frac{c^{o}}{c}-b} \hat{c}_{t-1}+\phi_{l} \hat{h}_{t}+\hat{\varepsilon}_{t}^{l} \\
& \widehat{M R S}_{t}^{r t}=\frac{\sigma}{1-b \frac{c}{c^{r t}}} \hat{c}_{t}^{r t}-\frac{b \sigma}{\frac{c^{r t}}{c}-b} \hat{c}_{t-1}+\phi_{l} \hat{h}_{t}+\hat{\varepsilon}_{t}^{l} \\
& \hat{u}_{t}+\hat{k}_{t}-\hat{h}_{t}-\hat{g}_{z, t}=\hat{w}_{t}-\hat{r}_{t}^{k} \\
& \widehat{m c}_{t}=-\hat{\varepsilon}_{t}^{a}+\alpha \hat{r}_{t}^{k}+(1-\alpha) \hat{w}_{t} \\
& \hat{y}_{t}=\frac{y+\Phi}{y}\left[\hat{\varepsilon}_{t}^{a}+\alpha\left(\hat{k}_{t}+\hat{u}_{t}\right)+(1-\alpha) \hat{h}_{t}\right] \\
& \hat{R}_{t}=\phi_{R} \hat{R}_{t-1}+\left(1-\phi_{R}\right)\left(\phi_{\pi} \hat{\pi}_{t}+\phi_{y}\left(\hat{y}_{t}-\hat{y}_{t}^{\text {flex }}\right)\right)+\phi_{\Delta y}\left(\hat{y}_{t}-\hat{y}_{t-1}-\left(\hat{y}_{t}^{\text {flex }}-\hat{y}_{t-1}^{\text {flex }}\right)\right)+\hat{\varepsilon}_{t}^{r}
\end{aligned}
$$




\section{A.3 Robustness}

Table A.1. Determinacy versus Indeterminacy - Alternative Prior for $\theta$ (1955Q4-1979Q2)

\begin{tabular}{ccccccc}
\hline \hline \multirow{2}{*}{ Region } & Prior for $\theta$ & \multicolumn{2}{c}{ Log-data density } & \multicolumn{2}{c}{ Probability } & KR ratio \\
\cline { 3 - 6 } & Determinacy & Indeterminacy & Determinacy & Indeterminacy & \\
\hline SADL & Uniform $(0,1)$ & -619.62 & -609.66 & 0 & 1 & 19.9 \\
IADL & Uniform $(0,1)$ & -702.59 & -705.28 & 0.94 & 0.06 & 5.4 \\
KR ratio & 165.9 & 95.6 & & & \\
\hline
\end{tabular}

Notes: The prior probability of determinacy is 0.52. SADL and IADL stand for standard aggregate demand logic and inverse aggregate demand logic, respectively. Log marginal data densities are approximated by Geweke's (1999) harmonic mean estimator. The posterior probabilities are calculated based on the output of the Metropolis algorithm. KR stands for Kass and Raftery.

Table A.2. Determinacy versus Indeterminacy - Taylor Rule with expected inflation (1955Q4-1979Q2)

\begin{tabular}{cccccc}
\hline \hline & & \multicolumn{2}{c}{ Log-data density } & \multicolumn{2}{c}{ Probability } \\
\cline { 3 - 6 } Region & Prior for $\theta$ & Determinacy & Indeterminacy & Determinacy & Indeterminacy \\
\hline SADL & Uniform $(0,1)$ & -620.59 & -609.06 & 0 & 1 \\
IADL & Uniform $(0,1)$ & -703.32 & -703.60 & 0.57 & 0.43 \\
RANK & - & -625.21 & -609.49 & 0 & 1 \\
\hline
\end{tabular}

Notes: The prior probability of determinacy is 0.52. SADL and IADL stand for standard aggregate demand logic and inverse aggregate demand logic, respectively. Log marginal data densities are approximated by Geweke's (1999) harmonic mean estimator. The posterior probabilities are calculated based on the output of the Metropolis algorithm. KR stands for Kass and Raftery. 


\section{A.4 Impulse response functions}
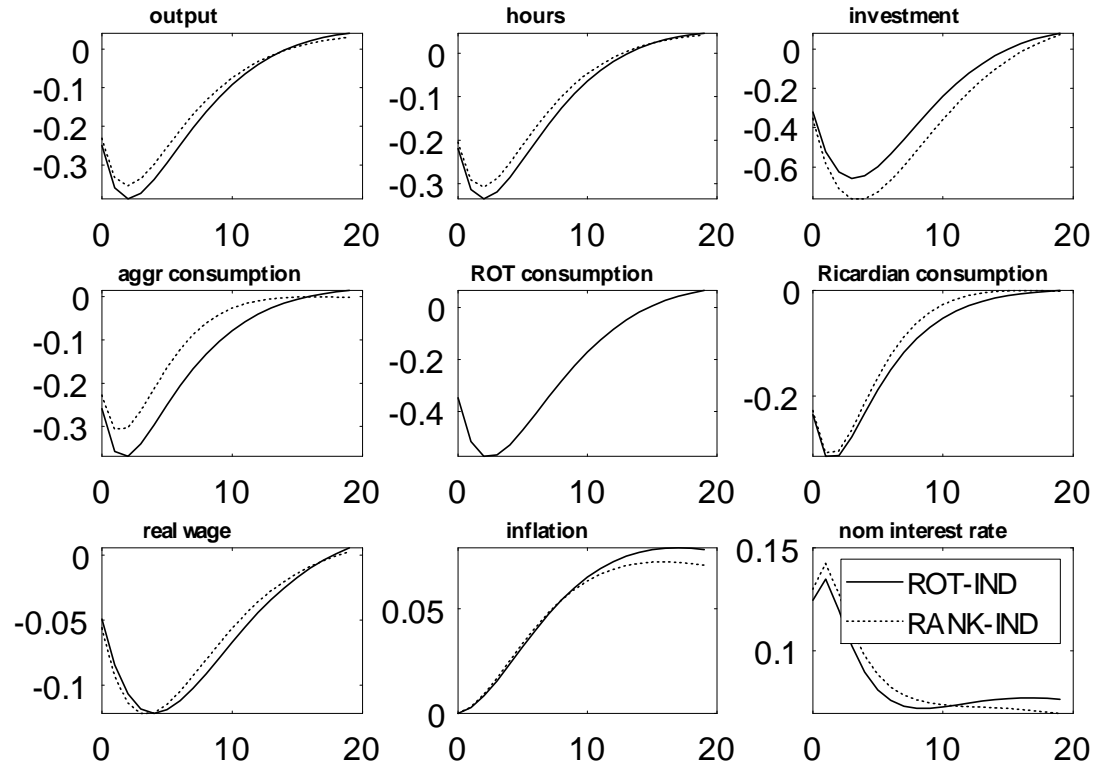

Figure A.1: Impulse responses to a one standard deviation monetary policy shock (Sample: 1955Q4-1979Q2)
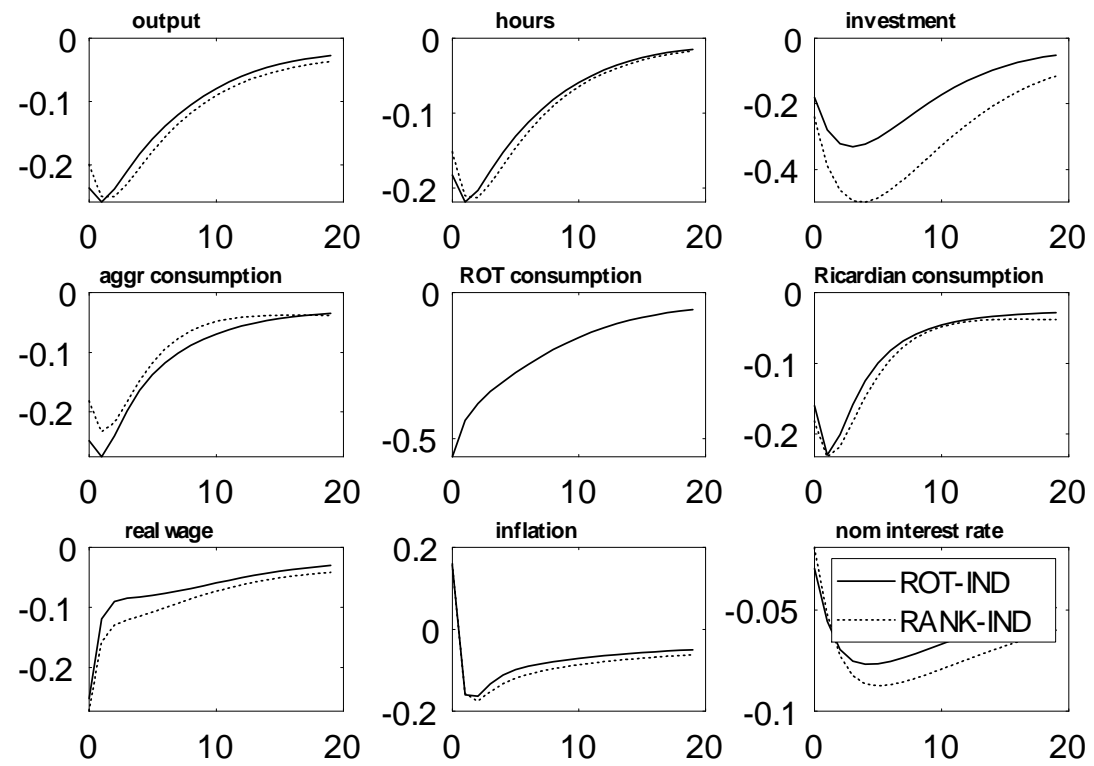

Figure A.2: Impulse responses to a one standard deviation price markup shock (Sample: 1955Q4-1979Q2) 

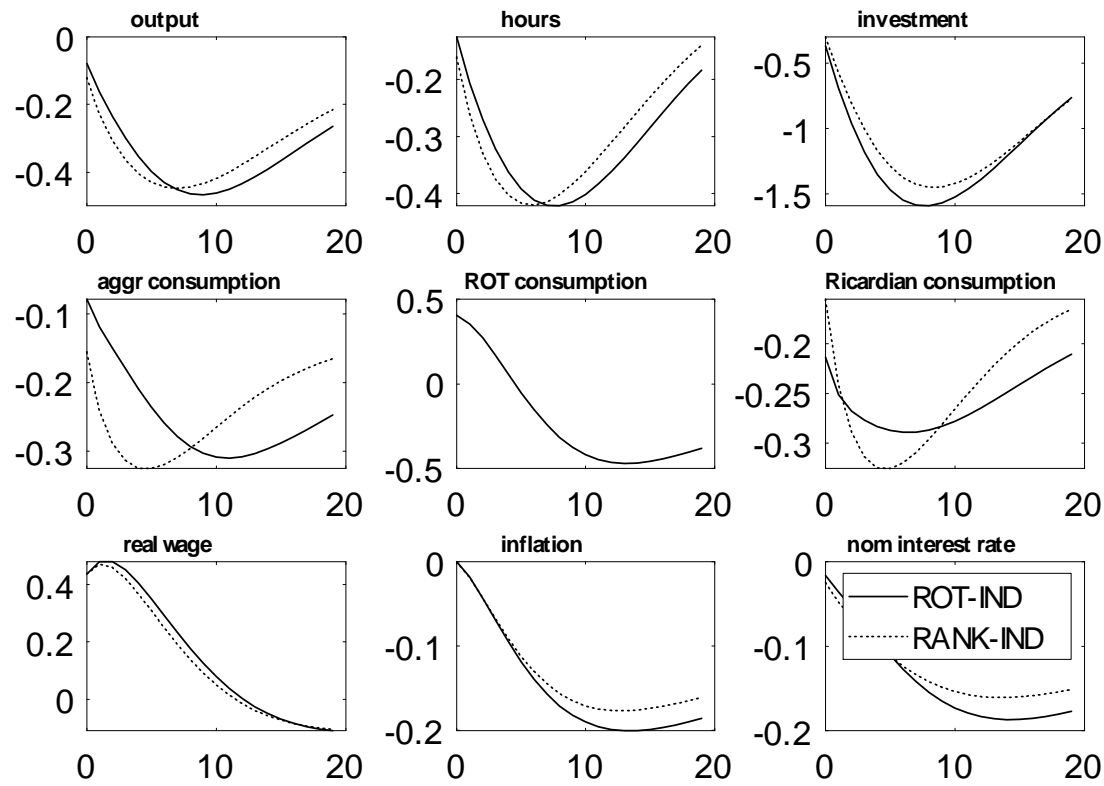

Figure A.3: Impulse responses to a one standard deviation wage markup shock (Sample: 1955Q4-1979Q2)
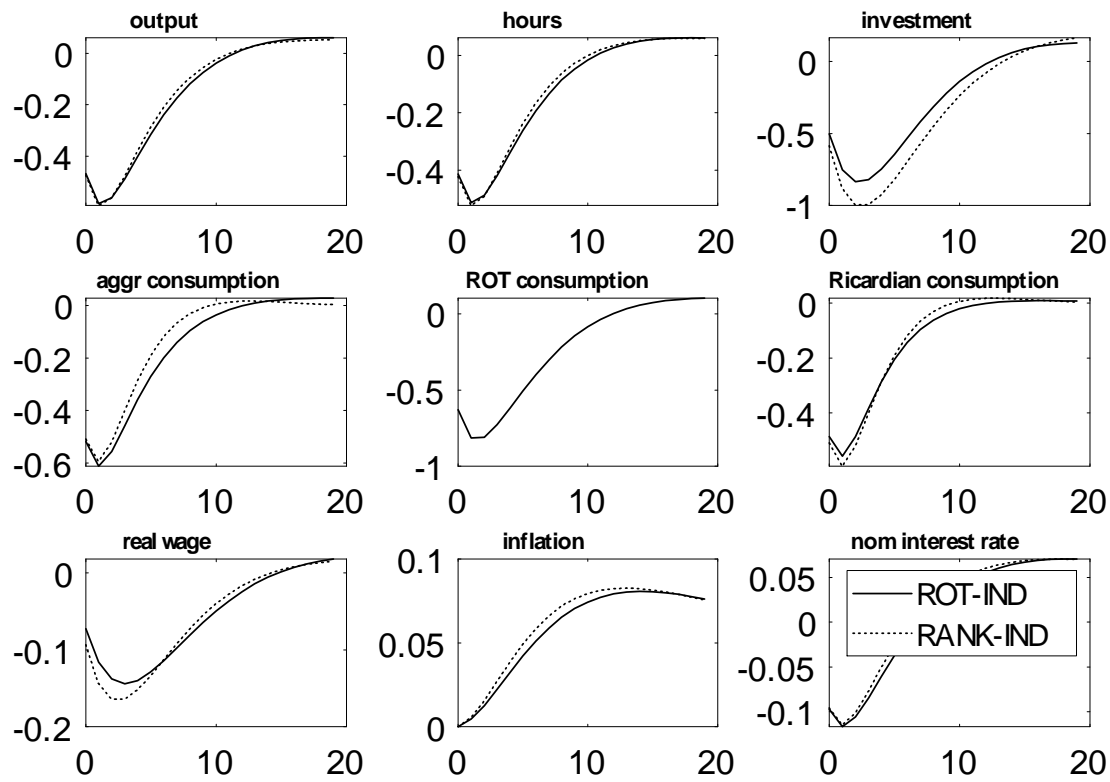

Figure A.4: Impulse responses to a one standard deviation risk premium shock (Sample: 1955Q4-1979Q2) 

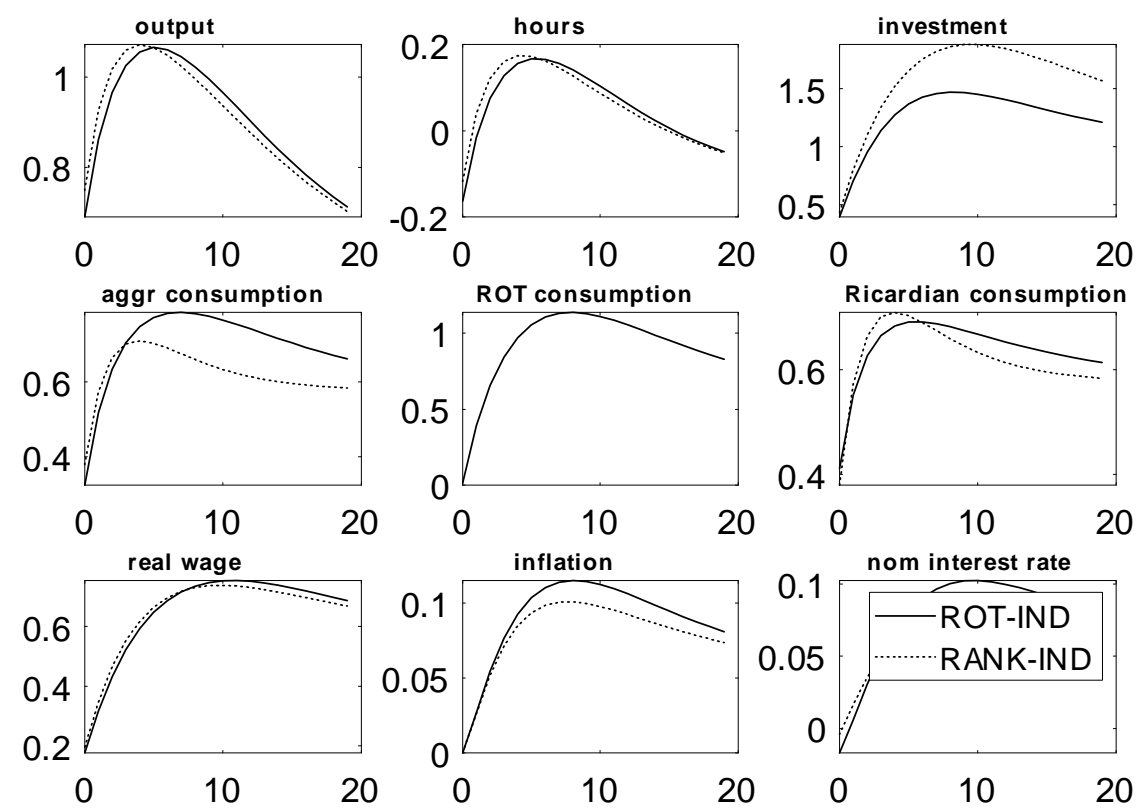

Figure A.5: Impulse responses to a one standard deviation technology shock (Sample: 1955Q41979Q2)
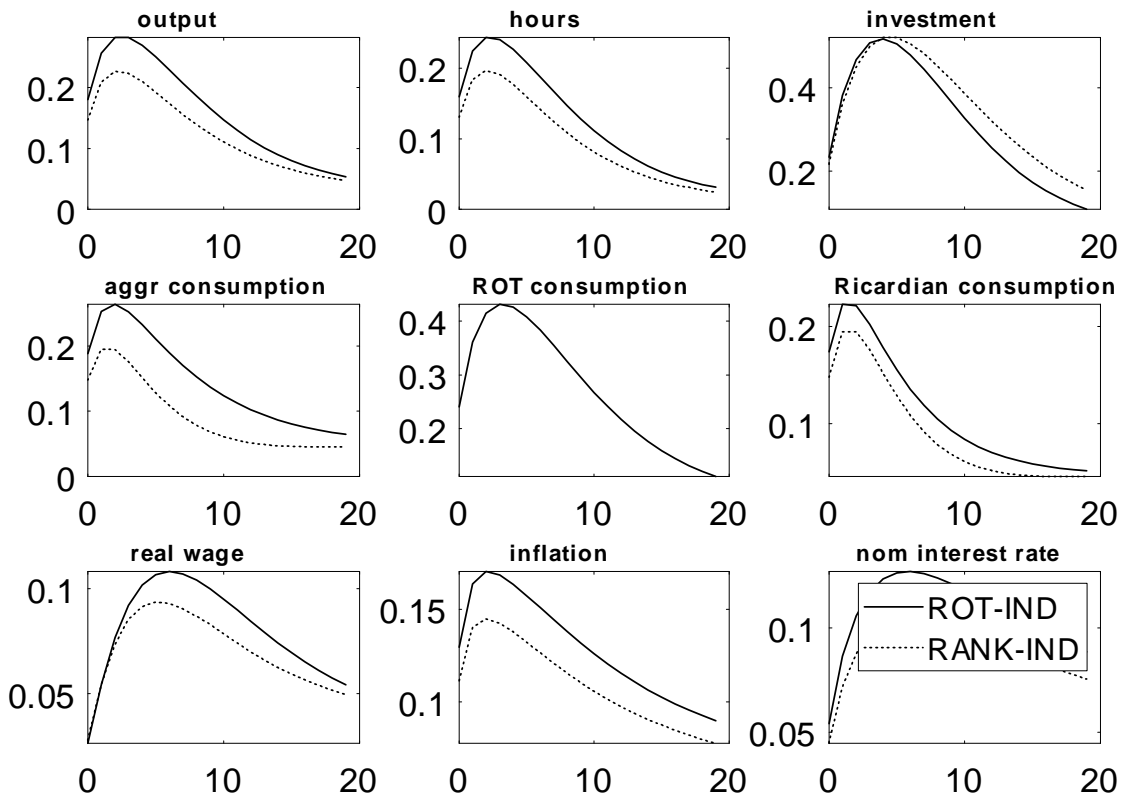

Figure A.6: Impulse responses to a one standard deviation sunspot shock (Sample: 1955Q41979Q2) 